\title{
Characterization of the size-segregated water-soluble inorganic ions at eight Canadian rural sites
}

\author{
L. Zhang, R. Vet, A. Wiebe, C. Mihele, B. Sukloff, E. Chan, M. D. Moran, and S. Iqbal \\ Air Quality Research Division, Science and Technology Branch, Environment Canada, 4905 Dufferin Street, Toronto, \\ Ontario, M3H 5T4, Canada
}

Received: 15 May 2008 - Published in Atmos. Chem. Phys. Discuss.: 21 July 2008

Revised: 4 November 2008 - Accepted: 4 November 2008 - Published: 9 December 2008

\begin{abstract}
Size-segregated water-soluble inorganic ions, including particulate sulphate $\left(\mathrm{SO}_{4}^{2-}\right)$, nitrate $\left(\mathrm{NO}_{3}^{-}\right)$, ammonium $\left(\mathrm{NH}_{4}^{+}\right)$, chloride $\left(\mathrm{Cl}^{-}\right)$, and base cations $\left(\mathrm{K}^{+}, \mathrm{Na}^{+}\right.$, $\mathrm{Mg}^{2+}, \mathrm{Ca}^{2+}$ ), were measured using a Micro-Orifice Uniform Deposit Impactor (MOUDI) during fourteen short-term field campaigns at eight locations in both polluted and remote regions of eastern and central Canada. The size distributions of $\mathrm{SO}_{4}^{2-}$ and $\mathrm{NH}_{4}^{+}$were unimodal, peaking at $0.3-0.6 \mu \mathrm{m}$ in diameter, during most of the campaigns, although a bimodal distribution was found during one campaign and a trimodal distribution was found during another campaign made at a coastal site. $\mathrm{SO}_{4}^{2-}$ peaked at slightly larger sizes in the cold seasons $(0.5-0.6 \mu \mathrm{m})$ compared to the hot seasons $(0.3-$ $0.4 \mu \mathrm{m}$ ) due to the higher relative humidity in the cold seasons. The size distributions of $\mathrm{NO}_{3}^{-}$were unimodal, peaking at $4.0-7.0 \mu \mathrm{m}$ during the warm-season campaigns, and bimodal, with one peak at $0.3-0.6 \mu \mathrm{m}$ and another at $4-7 \mu \mathrm{m}$ during the cold-season campaigns. A unimodal size distribution, peaking at 4-6 $\mu \mathrm{m}$, was found for $\mathrm{Cl}^{-}, \mathrm{Na}^{+}, \mathrm{Mg}^{2+}$, and $\mathrm{Ca}^{2+}$ during approximately half of the campaigns and a bimodal distribution, with one peak at $2 \mu \mathrm{m}$ and the other at $6 \mu \mathrm{m}$, was found during the rest of the campaigns. For $\mathrm{K}^{+}$, a bimodal distribution, with one peak at $0.3 \mu \mathrm{m}$ and the other at $4 \mu \mathrm{m}$, was observed during most of the campaigns. Seasonal contrasts in the size-distribution profiles suggest that emission sources and air mass origins were the major factors controlling the size distributions of the primary aerosols while meteorological conditions were more important for the secondary aerosols.

The dependence of the particle acidity on the particle size from the nucleation mode to the accumulation mode was not consistent from site to site or from season to season. Particles in the accumulation mode were more acidic than those in the
\end{abstract}

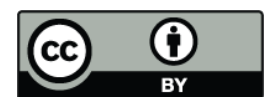

Correspondence to: L. Zhang

(leiming.zhang@ec.gc.ca) nucleation mode when submicron particles were in the state of strong acidity; however, when submicron particles were neutral or weakly acidic, particles in the nucleation mode could sometimes be more acidic. The inconsistency of the dependence of the particle acidity on the particle size should have been caused by the different emission sources of all the related species and the different meteorological conditions during the different campaigns. The results presented here at least partially explain the controversial phenomenon found in previous studies on this topic.

\section{Introduction}

In order to address the impacts of atmospheric aerosols on air-quality, ecosystem health, and climate change, it is essential to gain a thorough knowledge of their chemical composition and size distributions at high temporal resolution and at local, regional, and global scales (Andreae and Crutzen, 1997; McMurry, 2000; Kaufman et al., 2002; IPCC, 2007). For example, recently developed size-resolved air-quality and climate models all require size-distribution data of different aerosol species as inputs (e.g., Ackermann et al., 1998; Gong et al., 2006). Another example involving size-resolved atmospheric aerosols is acid deposition issues due to the strong dependence of particle dry deposition on particle size (Pryor et al., 2008). Acid deposition that exceeds the critical load of an ecological system will cause long-term harmful effects. Different ecosystems have different critical loads due to their different buffering capacities or sensitivities to acid deposition. Sulphur and nitrogen species contribute to acid deposition (Whepdale et al., 1997) while the deposition of base cations $\left(\mathrm{Ca}^{2+}, \mathrm{Mg}^{2+}, \mathrm{K}^{+}\right.$and $\left.\mathrm{Na}^{+}\right)$increases the alkalinity of the surface and thus the ecosystems' buffering capacity (Watmough et al., 2005; Skeffington, 2006; McNaulty et al., 2007). To assess the effects of acid deposition on sensitive ecosystems, the atmospheric deposition of both acidic

Published by Copernicus Publications on behalf of the European Geosciences Union. 
species and base cations needs to be estimated with sufficient accuracy (Environment Canada, 2005).

Substantial knowledge has been gained on the size distributions of the major water-soluble inorganic ions during the past four decades. Non-sea salt sulphate $\left(\mathrm{SO}_{4}^{2-}\right)$ and ammonium $\left(\mathrm{NH}_{4}^{+}\right)$were found to be predominantly in the fine particle mode while sea spray $\mathrm{SO}_{4}^{2-}, \mathrm{Cl}^{-}, \mathrm{Na}^{+}, \mathrm{Mg}^{2+}$, and $\mathrm{Ca}^{2+}$ were more abundant in the coarse fraction (Milford and Davidson, 1987; Hillamo et al., 1998; Heintzenberg et al., 2000; Parmar et al., 2001; Lestari et al., 2003; Park and Kim, 2004; Xiu et al., 2004; Tsai et al., 2005). Fine and coarse $\mathrm{NO}_{3}^{-}$were both important to its total mass, and their relative fractions were determined by the process through which they were formed, i.e., by the reaction of gaseous $\mathrm{HNO}_{3}$ with ammonia (fine) or with alkaline species in large particles (coarse) (Kadawaki, 1977; Wolff, 1984; Wall et al., 1988; Zhuang et al., 1999; Parmar et al., 2001; Lee et al., 2008). Many studies have found $\mathrm{K}^{+}$to be mostly in fine particles (Park and Kim, 2004; Park et al., 2004; Chen et al., 2005), although at some locations, coarse $\mathrm{K}^{+}$can be substantial (Krivácsy and Molnár, 1998). In some cases, a bimodal or trimodal distribution is needed to describe the size distribution of inorganic ions (Milford and Davidson, 1987; Zhuang et al., 1999; Lestari et al., 2003). Apparently, the particle size distributions vary greatly with season, location, and air-mass origin (Birmili et al., 2001; Hazi et al., 2003; Tunved et al., 2003; Park et al., 2004; Trebs et al., 2004; Van Dingenen et al., 2005; Abdalmogith and Harrison, 2006; Fisseha et al., 2006).

Despite some field studies conducted at various locations in eastern North America measuring the chemical composition of size-resolved particles (e.g., Hazi et al., 2003; Rupakheti et al., 2005; Lee et al., 2008), there is still a lack of understanding of the size distribution profiles of many particle species in these regions, especially at remote locations in northern Canada where there are no major nearby emission sources. During 2001-2005, fourteen short-term field campaigns were conducted by Environment Canada to measure the size-segregated water-soluble inorganic ions at eight rural and remote sites, which are thought to represent the regional background air in their respective locations. Back trajectory analysis has been conducted to identify the effects of air-mass origins on the seasonal and geographical patterns of the measured ion mass concentrations and size-distribution profiles. Discussion on the size-dependent particle acidity, based on the charge-equivalent cation/anion ratios, is also presented here.

Although the primary goal of the present study is to characterize the size distributions of the background inorganic ions over eastern Canada for improving our future acid deposition, air-quality and climate modelling, and health studies, the results should be useful for applications elsewhere. This is because the physical and chemical mechanisms of the formation of the inorganic ions and the dependence of their size distributions on the meteorological conditions are similar everywhere around the world. Since the current study includes both the cold and the warm seasons, and was conducted at sites located in both polluted and remote regions, the seasonal and geographical patterns of the observed size distributions and the size-dependent particle acidity will provide valuable information to other regions where only limited data are available.

\section{Experimental design and method}

\subsection{Emission sources and measurement sites}

The ion species measured in the fourteen campaigns included $\mathrm{SO}_{4}^{2-}, \mathrm{NO}_{3}^{-}, \mathrm{NH}_{4}^{+}, \mathrm{Cl}^{-}, \mathrm{K}^{+}, \mathrm{Na}^{+}, \mathrm{Mg}^{2+}$, and $\mathrm{Ca}^{2+}$. The majority of $\mathrm{SO}_{4}^{2-}, \mathrm{NO}_{3}^{-}$, and $\mathrm{NH}_{4}^{+}$in this region exist as secondary particles, i.e., formed from their gaseous precursors $\left(\mathrm{SO}_{2}, \mathrm{NO}_{\mathrm{x}}\right.$, and $\mathrm{NH}_{3}$, respectively) through various chemical reactions (Vet et al., 2001). $\mathrm{Mg}^{2+}$ and $\mathrm{Ca}^{2+}$ are mainly from soil dust emissions, $\mathrm{Cl}^{-}$and $\mathrm{Na}^{+}$from sea salt and road salt emissions, and $\mathrm{K}^{+}$from soil dust emissions and from biomass burning and vegetation. In order to understand the geographical patterns of the observed ion distributions, emission distributions of the precursors have to be known. Emissions of $\mathrm{SO}_{2}, \mathrm{NO}_{\mathrm{x}}, \mathrm{NH}_{3}$, and $\mathrm{PM}_{10}$ (particles with aerodynamic diameter smaller than $10 \mu \mathrm{m}$ ) combining year 2000 Canadian sources and year 2001 USA sources have been aggregated into the Canadian air-quality model grids $(\sim 42 \mathrm{~km}$ by $42 \mathrm{~km}$ ) and are shown in Fig. 1. In Canada, southern Ontario and southern Quebec have high emissions of $\mathrm{SO}_{2}$, $\mathrm{NO}_{\mathrm{x}}, \mathrm{NH}_{3}$, and $\mathrm{PM}_{10}$ since these areas have a high population density, many industries, and intense agricultural activities. The southern Prairies of western Canada also have quite high emissions of $\mathrm{SO}_{2}, \mathrm{NO}_{\mathrm{x}}, \mathrm{NH}_{3}$, and $\mathrm{PM}_{10}$ due to downstream oil and gas production and agricultural activities. In the USA, most parts of the eastern half of the USA have high emissions of $\mathrm{SO}_{2}, \mathrm{NO}_{\mathrm{x}}, \mathrm{NH}_{3}$, and $\mathrm{PM}_{10}$; however, the highest emissions of $\mathrm{SO}_{2}$ and $\mathrm{NO}_{\mathrm{x}}$ are located in the eastern half of the US Midwest and the Mid-Atlantic, the highest $\mathrm{NH}_{3}$ emissions are located in the central area of the US Midwest (the Great Plains), and the highest $\mathrm{PM}_{10}$ emissions are located in most parts of the US Midwest. Seasonal variations of emissions cannot be shown in Fig. 1 and they are briefly discussed wherever needed in Sect. 3.

Eight sites were selected to conduct the field studies, seven of which were located in eastern Canada and one in central Canada (see the top-left map in Fig. 2). All these sites are ground level Canadian Air and Precipitation Monitoring Network sites (CAPMoN). The selected sites covered a large area of eastern Canada, including both polluted and relatively remote regions. Comparing Fig. 2 with Fig. 1, three sites (EGB, FRS, and BRL) can be defined as located in polluted regions, three (ALG, CHA, and SPR) in less polluted regions, and two (KEJ and LED) in relatively clean regions. 

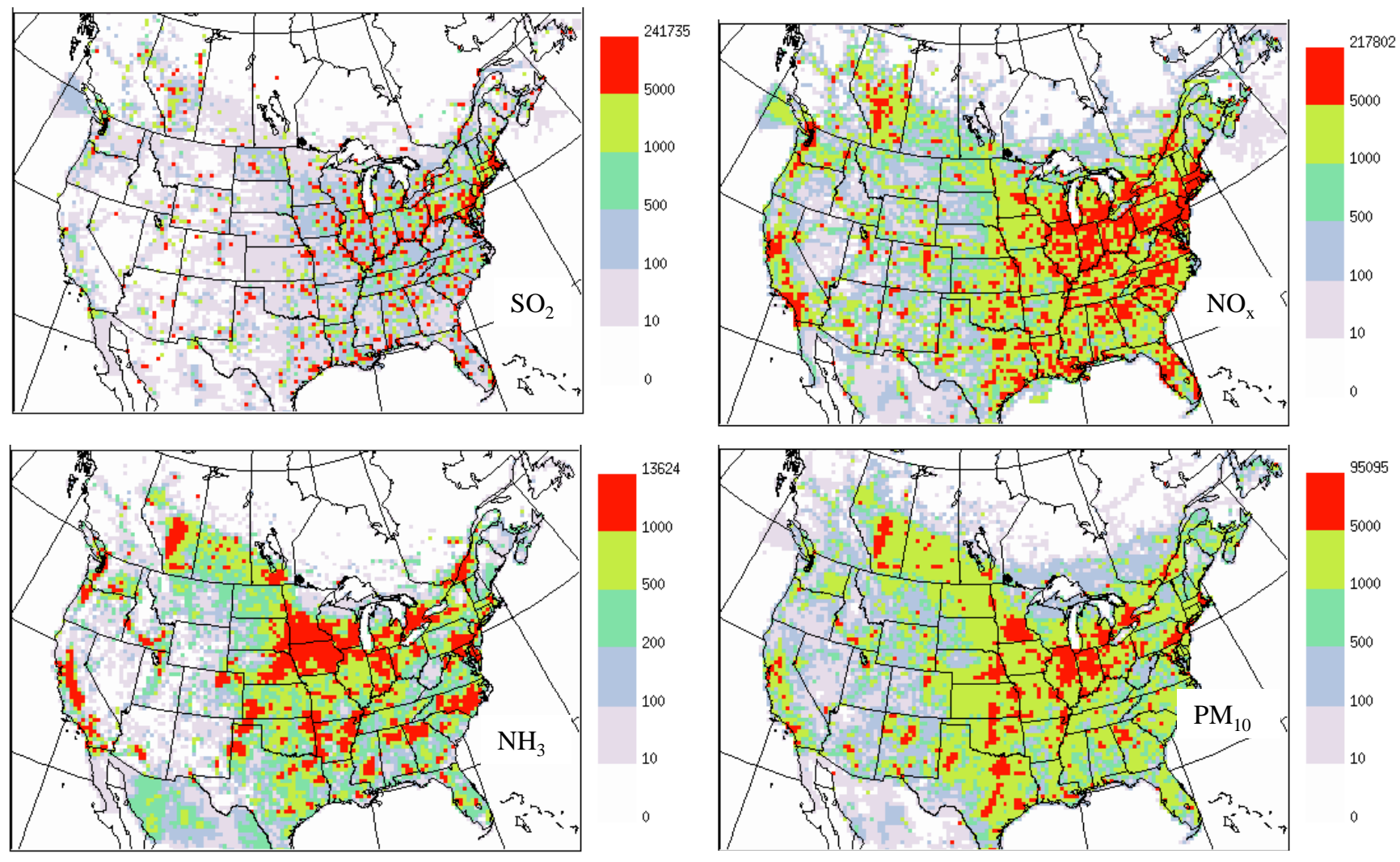

Fig. 1. Maps of emission sources of $\mathrm{SO}_{2}, \mathrm{NO}_{\mathrm{x}}$ (in the equivalent mass of $\mathrm{NO}_{2}$ ), $\mathrm{NH}_{3}$ and $\mathrm{PM}_{10}$ based on $2000 \mathrm{Canadian}$ and $2001 \mathrm{US}$ emission inventory (the unit is tonnes/grid/year with the grid size of $\sim 42 \mathrm{~km}$ by $42 \mathrm{~km}$ ).

However, long-range transport is known to transport pollutants from the polluted regions to the remote locations, as will be discussed in Sect. 3 .

\subsection{Measurement periods}

Table 1 lists the time periods when the field campaigns were conducted. Since different sites are located at different latitudes and some sites are affected by ocean air masses, the traditionally-defined seasons (based on the month of the year) might not be consistent from site to site. Thus, the average daytime (09:00-17:00 local time) air temperature (see Table 1) was used to define the cold-, warm- and hotseason campaigns. Seven campaigns (FRS1, EGB1, ALG1, LED2, CHA2, SPR2, BRL1) were defined as cold-season campaigns, two campaigns (FRS2, KEJ2) were defined as warm-season campaigns, and five campaigns (KEJ1, ALG2, LED1, CHA2, SPR1) were defined as hot-season campaigns. Note that at the three polluted sites, three campaigns were carried out during the cold season (FRS1, EGB1, BRL1) and one during the warm season (FRS2); at the three less polluted sites, three campaigns were carried out during the cold season (ALG1, CHA1, SPR2) and three during the hot season (ALG2, CHA2, SPR1); and at the two clean sites, one campaign was carried out during the cold season (LED2), one during the warm season (KEJ2), and two during the hot season (LED1, KEJ1).

\subsection{Sample and analysis}

Air samples were collected using a Micro-Orifice Uniform Deposit Impactor sampler (MOUDI Model 110, MSN Minneapolis, MN, USA) at a mass flow rate of $30 \mathrm{~L} / \mathrm{min}$ at $0^{\circ} \mathrm{C}$ and $1 \mathrm{~atm}$. The sampler was run with 11 fractionation stages with the following 50\% cut-off points for the particle aerodynamic diameters: $18,9.9,6.2,3.1,1.8,1.0,0.54,0.32$, $0.18,0.093$, and $0.048 \mu \mathrm{m}$, followed by a backup filter. The MOUDI sampler was located $5 \mathrm{~m}$ above ground level under a rain shelter that allowed for free ventilation. Teflon ${ }^{\circledR}$ filters of $47 \mathrm{~mm}$ diameter and $0.1 \mathrm{~mm}$ thickness (PTFE, Savillex Corporation, Minnetonka, Minnesota) were used in all stages. After sampling, the filters were stored in pre-washed $10 \mathrm{~mL}$ plastic vials at $5-10^{\circ} \mathrm{C}$.

Blank values were determined from filters that were loaded into a MOUDI sampler and then unloaded after $24 \mathrm{~h}$ of inactive sampling near the same location as the active sampling. This duplicated all handling procedures for both the active and blank filters. Standard ion chromatography (Dionex Corporation, Sunnyvale, California) was used for analyses of all filters after extraction in deionized water. Average field blank values were calculated by replacing all 


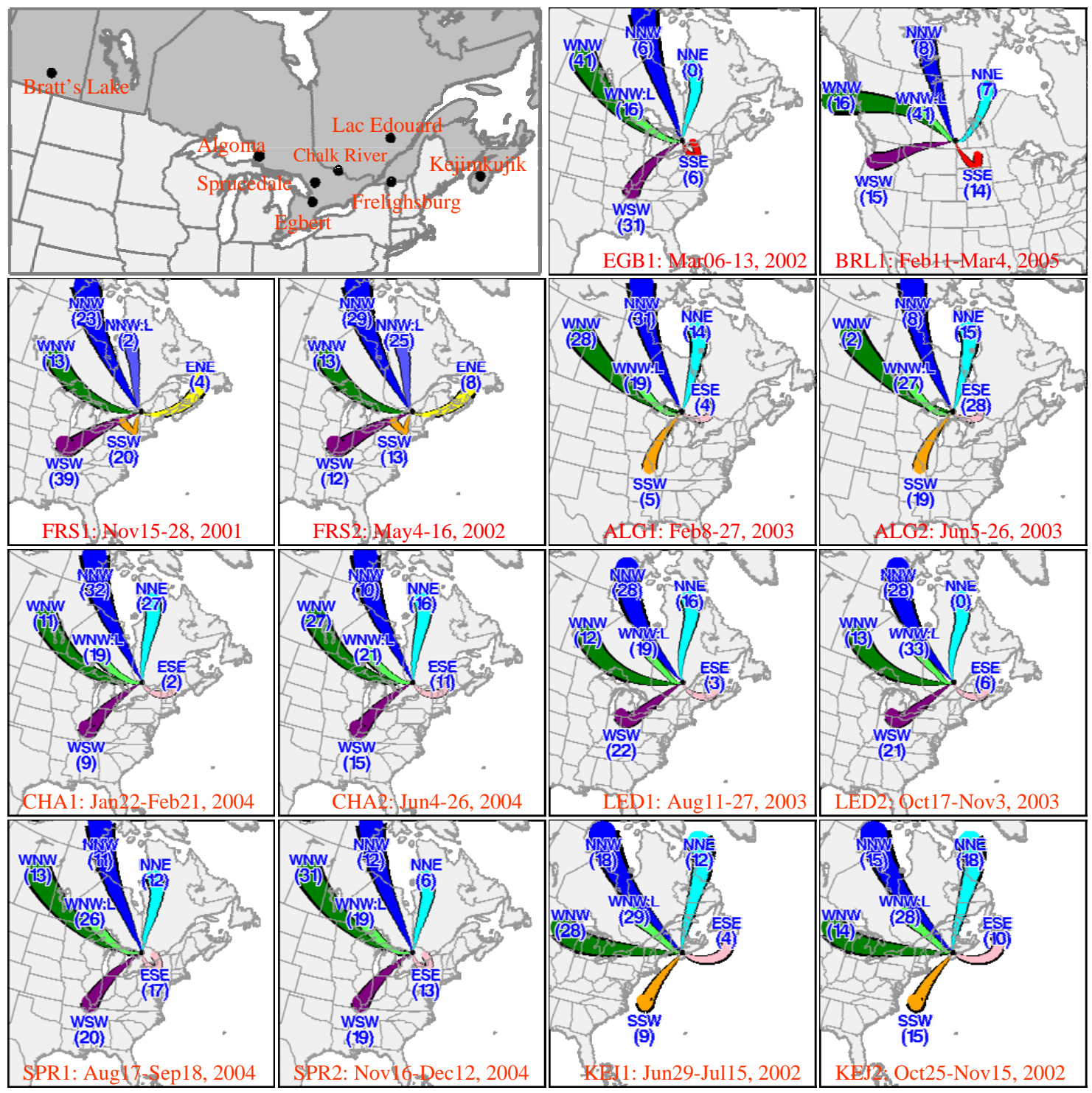

Fig. 2. Locations of the eight measurement sites and the six trajectory clusters at these sites defined from the average of 2001-2005 three-day six-hour back trajectories. Number in the bracket positioned at the end of each cluster represents the percentage of all trajectories associated with that cluster during the specific campaign period.

negative and "no response" values from the laboratory with $2 / 3$ of their reported analytical detection limit (BDL). The BDL was described as 3 standard deviations of repeated measurements of a quality control solution at about 40 parts per trillion (ppt). Air concentrations for each aerosol species were then calculated as: $C_{i}=\left(M_{i}-B V_{i}\right) /(M F R * E t)$, where $C_{i}, M_{i}$ and $B V_{i}$ are concentration, collected mass and blank value, respectively, of species $i$ during one sample period $(E t)$ and $M F R$ is the mass flow rate (so $M F R * E t$ represents sampled air volume). The sampling duration $(E t)$ ranged from 6 to $152 \mathrm{~h}$ (Table 1) depending on the perceived concentration levels at the time of sampling. The number of samples during each campaign is listed in Table 1.

\subsection{Quality of MOUDI data}

All samples were plotted for data quality control purposes. It was not uncommon that concentrations were lower than the 3-times standard deviation of all blanks divided by the mean air volume sampled (3SDB). Under such circumstances, the data were not quantitative due to a large noise-to-signal problem. For each ion species and for every campaign, the percentage of samples having a concentration lower than blank values (3SDB) was calculated. When calculating these percentages, only samples from stages 3-10 (mid-point size 6.2 to $0.093 \mu \mathrm{m}$ ) were used for $\mathrm{SO}_{4}^{2-}, \mathrm{NO}_{3}^{-}, \mathrm{NH}_{3}$, and $\mathrm{K}^{+}$because the other stages, i.e., the first two and the last two, had very low concentrations of these species; similarly, only 
Table 1. List of 14 field campaigns.

\begin{tabular}{|c|c|c|c|c|c|c|c|}
\hline Campaign & Site & Lat $\mathrm{N}$, long $\mathrm{W}$ & Campaign period & $\begin{array}{l}\text { Mean daytime } \\
\text { temperature } \\
\left({ }^{\circ} \mathrm{C}\right)\end{array}$ & $\begin{array}{l}\text { Hourly } \\
\text { average } \\
\text { RH (\%) }\end{array}$ & $\begin{array}{l}\text { Number } \\
\text { of } \\
\text { Samples }\end{array}$ & $\begin{array}{l}\text { Sample } \\
\text { duration } \\
\text { (h) }\end{array}$ \\
\hline FRS1 & Frelighsberg, Quebec & $45.05,73.06$ & 15-28 Nov 2001 & 7 & & 17 & $6-25$ \\
\hline FRS2 & & & 4-16 May 2002 & 12 & 64 & 7 & $11-48$ \\
\hline EGB1 & Egbert, Ontario & $44.23,79.78$ & 6-13 Mar 2002 & 2 & 86 & 8 & $6-16$ \\
\hline KEJ1 & Kejimkujik, Nova Scotia & $44.43,65.21$ & 29 Jun-15 Jul 2002 & 23 & 76 & 17 & $8-47$ \\
\hline KEJ2 & & & 25 Oct-15 Nov 2002 & 6 & 86 & 20 & $13-25$ \\
\hline ALG1 & Algoma, Ontario & $47.04,84.38$ & 8-27 Feb 2003 & -13 & 72 & 13 & $19-71$ \\
\hline ALG2 & & & 5-26 Jun 2003 & 22 & 59 & 24 & $10-50$ \\
\hline LED1 & Lac Edouard, Quebec & $47.68,72.44$ & 11-27 Aug 2003 & 19 & 83 & 12 & $24-48$ \\
\hline LED2 & & & 17 Oct-3 Nov 2003 & 2 & 87 & 9 & $21-72$ \\
\hline CHA1 & Chalk River, Ontario & $46.06,77.40$ & 22 Jan-21 Feb 2004 & -10 & 65 & 14 & $8-127$ \\
\hline CHA2 & & & 4-26 Jun 2004 & 19 & 68 & 11 & 24-119 \\
\hline SPR1 & Sprucedale, Ontario & $45.42,79.49$ & 17 Aug-18 Sep 2004 & 19 & 79 & 17 & 24-96 \\
\hline SPR2 & & & 16 Nov-12 Dec 2004 & 0 & 90 & 12 & $13-152$ \\
\hline BRL1 & Bratt's Lake, Saskatchewan & $50.20,104.20$ & 11 Feb-4 Mar 2005 & -8 & 93 & 11 & 21-106 \\
\hline
\end{tabular}

samples from stages 1-8 (sizes larger than $0.32 \mu \mathrm{m}$ ) were used for the other four species. The data were grouped into four data-quality categories based on these percentage numbers and based on scrutiny of the size-distribution plots. This is because, under certain circumstances, a clear peak in the size distribution was evident from all samples, yet the percentage of samples having concentrations higher than blank values (3SDB) was very low due to the extremely low concentrations in the stages not corresponding to the peak sizes. Under this scenario, the data were believed to represent the real size distribution profiles. The percentage numbers (using samples from MOUDI stages 3-10 for $\mathrm{SO}_{4}^{2-}, \mathrm{NO}_{3}^{-}, \mathrm{NH}_{3}$, and $\mathrm{K}^{+}$and stages 1-8 for the other four species) and the defined data quality category are all shown in Table 2.

For $\mathrm{SO}_{4}^{2-}, \mathrm{NO}_{3}^{-}$, and $\mathrm{NH}_{4}^{+}, 90-100 \%, 80-90 \%$, and $<80 \%$ of data from categories 1,2 , and 3, respectively, had concentrations higher than their respective blank values (3SDB). No category 4 data were identified for these three ion species. For the other five species, $85-100 \%$ and $50-85 \%$ of categories 1 and 2 data, respectively, had concentrations higher than their respective blank values (3SDB) values; the rest of the data were identified as categories 3 and 4, based mainly on the plotted size-distribution profiles. Data belonging to categories 1 and 2 were thought to be reliable while data belonging to categories 3 and 4 were less reliable. Note that errors caused by chemical transformation (e.g., $\mathrm{NH}_{4} \mathrm{NO}_{3}$ volatility) during the sampling process were not considered in this approach. As a short summary, data for $\mathrm{SO}_{4}^{2-}$ and $\mathrm{NH}_{4}^{+}$during all fourteen campaigns and data for $\mathrm{NO}_{3}^{-}$during most of the campaigns were of good quality in categories 1 and 2. For the rest of the species, the data quality ranged from relatively good (category 2) to very poor (category 4) depending on the nature of the site and the time of the year.

\subsection{Back trajectory cluster analysis}

In order to identify the source regions of the sampled aerosols and to better explain the geographical and seasonal patterns of the observed concentrations and size distributions, back trajectory cluster analyses, similar to the approach used in many previous studies (Abdalmogith and Harrison, 2005, and references therein), were conducted. Cluster analysis can account for variations in transport speed and direction simultaneously, yielding clusters of trajectories having similar length and curvature. Three-day back trajectories (every six hours) created by the Canadian Meteorological Centre for the period of 2001-2005 were run through a K-means clustering technique for each site. They were organized into six common clusters based on the commonality of Euclidian distance from trajectory to trajectory (Dorling, 1992). Figure 2 shows the six clusters at the eight sites where the fourteen campaigns were conducted.

The correlations between the ion concentrations and the pre-defined trajectory clusters can be explored by comparing the distributions of ion concentrations associated with different trajectory clusters. Since the samples collected in this study had variable durations and some samples had a very long duration (up to six days, see Table 1), and the campaigns covered only short periods of several weeks, it is difficult to assign the measured concentrations of individual samples to different trajectory clusters. Thus, we used five years of CAPMoN 24-h bulk (not size-resolved) concentrations to identify the dependence of the ion concentrations on the defined trajectory clusters. Note that there were four trajectories every day and CAPMoN monitored concentrations daily. Thus, each and every daily concentration was assigned to whatever clusters were associated with the four daily trajectories. The mean and median concentrations of the eight 
Table 2. Percentage of data from selected size stages (3-10 stages for $\mathrm{SO}_{4}^{2-}, \mathrm{NH}_{4}^{+}, \mathrm{NO}_{3}^{-}$and $\mathrm{K}^{+}$and $1-8$ stages for $\mathrm{Cl}^{-}, \mathrm{Na}^{+}, \mathrm{Mg}^{2+}$ and $\mathrm{Ca}^{2+}$ ) having concentrations higher than their respective 3SDB ( 3 times standard deviation of all blanks divided by the mean sample volume) and defined data-quality categories for all particle species during 14 campaigns (1 represents most reliable and 4 represents least reliable).

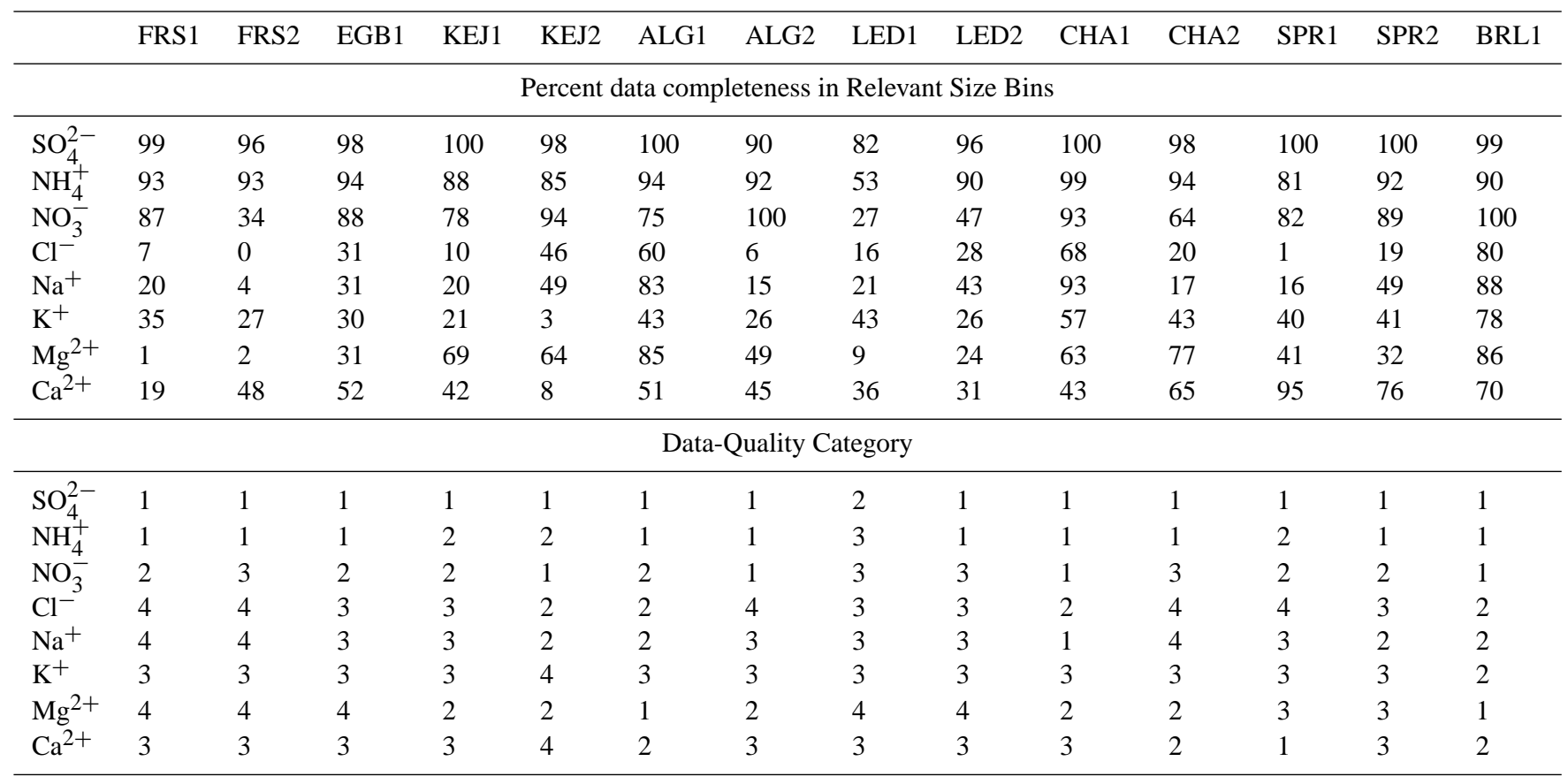

ion species for each trajectory cluster were generated from the 2001-2005 CAPMoN data (Table 3).

It can be seen from Table 3 that the highest concentrations of $\mathrm{SO}_{4}^{2-}, \mathrm{NO}_{3}^{-}$, and $\mathrm{NH}_{4}^{+}$were associated with clusters SSW at Algoma, SSE and WNWL at Bratt's Lake, WSW at Chalk River, SSE and WSW at Egbert, WSW at Frelighsburg, WNW at Kejimkujik, WSW at Lac Edouard, and WSW Sprucedale. Air masses associated with NNE and NNW had the lowest concentrations of $\mathrm{SO}_{4}^{2-}, \mathrm{NO}_{3}^{-}$, and $\mathrm{NH}_{4}^{+}$at Algoma, Chalk River, Egbert, Kejimkujik, Lac Edouard, and Sprucedale. At many locations (e.g., ALG, BRL, CHA, KEJ, LED and SPR), the clusters associated with the highest concentrations of $\mathrm{SO}_{4}^{2-}, \mathrm{NO}_{3}^{-}$, and $\mathrm{NH}_{4}^{+}$were also associated with the highest concentrations of $\mathrm{K}^{+}, \mathrm{Mg}^{2+}$, and $\mathrm{Ca}^{2+}$ due to the similar geographical patterns of the emissions of $\mathrm{SO}_{2}$, $\mathrm{NO}_{\mathrm{x}}, \mathrm{NH}_{3}$, and $\mathrm{PM}_{10}$ at these locations.

The 5-year average concentrations showed clear geographical patterns for most species. For example, EGB, FRS, SPR, and ALG had higher $\mathrm{SO}_{4}^{2-}$ concentrations compared to the rest of the sites, while EGB, FRS, and BRL had higher concentrations of $\mathrm{NO}_{3}^{-}, \mathrm{NH}_{4}^{+}$, and $\mathrm{Ca}^{2+}$, and $\mathrm{KEJ}$ and FRS had higher concentrations of $\mathrm{Cl}^{-}$and $\mathrm{Na}^{+}$. Geographical patterns of $\mathrm{K}^{+}$and $\mathrm{Mg}^{2+}$ were not apparent due to the very low concentrations of these two species, although BRL showed the highest concentrations.
It is known that the slowest (i.e., shortest) trajectory clusters passing over high emission areas will have the highest pollutant concentrations and the fastest clusters from clean areas will have the lowest concentrations (Hazi et al., 2003). Results shown in Table 3 support this theory, when comparing the defined trajectory clusters shown in Fig. 2 with the emission distributions shown in Fig. 1. The information provided above will be used to support the discussions presented in Sect. 3.

\section{Results}

Short- and long-range transport of emissions, meteorological conditions (temperature, relative humidity, precipitation, solar radiation/cloudiness, mixing layer height, etc), and physical and chemical mechanisms from which aerosols are produced are important factors relating to aerosol concentration levels and size distributions. In this section, we discuss in detail the seasonal and geographical patterns of the observed aerosol concentrations and size distributions and the possible causes of these patterns. For every ion species (Sects. 3.1$3.5)$, the following discussions are presented in sequence: (1) the production mechanism for each ion, (2) the seasonal contrast and associated causes of the campaign-average 
Table 3. Mean and median (left and right column separated by ',') atmospheric concentrations $\left(\mu \mathrm{g} \mathrm{m}^{-3}\right)$ of $\mathrm{SO}_{4}^{2-}, \mathrm{NO}_{3}^{-}, \mathrm{NH}_{4}^{+}, \mathrm{Cl}^{-}, \mathrm{Na}^{+}$, $\mathrm{K}^{+}, \mathrm{Mg}^{2+}$ and $\mathrm{Ca}^{2+}$ generated from CAPMoN monitored data during the period of 2001-2005.

\begin{tabular}{|c|c|c|c|c|c|c|c|c|c|}
\hline Site & Cluster & $\mathrm{SO}_{4}^{2-}$ & $\mathrm{NO}_{3}^{-}$ & $\mathrm{NH}_{4}^{+}$ & $\mathrm{Cl}^{-}$ & $\mathrm{Na}^{+}$ & $\mathrm{K}^{+}$ & $\mathrm{Mg}^{2+}$ & $\mathrm{Ca}^{2+}$ \\
\hline \multirow[t]{6}{*}{ ALG } & ESE & $2.29,1.19$ & $0.33,0.11$ & $0.70,0.34$ & $0.01,0.00$ & $0.04,0.02$ & $0.04,0.03$ & $0.03,0.01$ & $0.12,0.04$ \\
\hline & NNE & $0.72,0.58$ & $0.22,0.10$ & $0.22,0.14$ & $0.02,0.01$ & $0.05,0.03$ & $0.02,0.02$ & $0.02,0.01$ & $0.06,0.03$ \\
\hline & NNW & $0.76,0.66$ & $0.30,0.11$ & $0.23,0.13$ & $0.04,0.01$ & $0.07,0.04$ & $0.02,0.02$ & $0.03,0.01$ & $0.08,0.03$ \\
\hline & SSW & $4.04,2.60$ & $1.32,0.41$ & $1.54,1.12$ & $0.02,0.01$ & $0.05,0.02$ & $0.07,0.06$ & $0.05,0.02$ & $0.25,0.11$ \\
\hline & WNW & $1.10,0.91$ & $0.70,0.17$ & $0.47,0.27$ & $0.02,0.00$ & $0.05,0.02$ & $0.03,0.02$ & $0.03,0.02$ & $0.12,0.06$ \\
\hline & WNW:L & $1.69,1.20$ & $0.61,0.16$ & $0.61,0.34$ & $0.02,0.01$ & $0.04,0.02$ & $0.04,0.03$ & $0.03,0.02$ & $0.15,0.06$ \\
\hline \multirow[t]{6}{*}{ BRL } & NNE & $1.23,1.05$ & $0.83,0.56$ & $0.56,0.47$ & $0.04,0.01$ & $0.05,0.03$ & $0.07,0.05$ & $0.12,0.07$ & $0.44,0.23$ \\
\hline & NNW & $0.85,0.74$ & $0.74,0.49$ & $0.40,0.29$ & $0.06,0.02$ & $0.06,0.04$ & $0.10,0.05$ & $0.11,0.06$ & $0.40,0.21$ \\
\hline & SSE & $1.55,1.29$ & $1.22,0.70$ & $0.71,0.50$ & $0.03,0.02$ & $0.08,0.03$ & $0.12,0.07$ & $0.17,0.10$ & $0.67,0.40$ \\
\hline & WNW & $0.75,0.56$ & $0.92,0.54$ & $0.39,0.26$ & $0.05,0.03$ & $0.09,0.03$ & $0.10,0.06$ & $0.12,0.06$ & $0.45,0.25$ \\
\hline & WNW:L & $1.31,1.11$ & $1.27,0.77$ & $0.68,0.50$ & $0.04,0.02$ & $0.07,0.04$ & $0.10,0.07$ & $0.14,0.07$ & $0.51,0.26$ \\
\hline & WSW & $0.92,0.63$ & $1.30,0.68$ & $0.55,0.29$ & $0.05,0.03$ & $0.10,0.04$ & $0.09,0.06$ & $0.10,0.06$ & $0.41,0.24$ \\
\hline \multirow[t]{6}{*}{ CHA } & ESE & $2.30,1.48$ & $0.41,0.13$ & $0.72,0.43$ & $0.02,0.01$ & $0.07,0.03$ & $0.06,0.05$ & $0.02,0.02$ & $0.12,0.05$ \\
\hline & NNE & $0.85,0.63$ & $0.25,0.10$ & $0.24,0.13$ & $0.04,0.01$ & $0.08,0.05$ & $0.03,0.02$ & $0.02,0.01$ & $0.08,0.03$ \\
\hline & NNW & $0.87,0.73$ & $0.36,0.14$ & $0.27,0.15$ & $0.08,0.01$ & $0.11,0.05$ & $0.03,0.02$ & $0.03,0.02$ & $0.09,0.04$ \\
\hline & WNW & $1.50,1.13$ & $0.68,0.19$ & $0.59,0.33$ & $0.05,0.01$ & $0.08,0.03$ & $0.04,0.04$ & $0.03,0.02$ & $0.11,0.06$ \\
\hline & WNW:L & $2.14,1.34$ & $0.46,0.16$ & $0.69,0.38$ & $0.03,0.01$ & $0.07,0.03$ & $0.05,0.04$ & $0.03,0.02$ & $0.13,0.06$ \\
\hline & WSW & $3.70,2.46$ & $0.92,0.26$ & $1.32,1.02$ & $0.03,0.01$ & $0.06,0.03$ & $0.08,0.06$ & $0.03,0.02$ & $0.15,0.07$ \\
\hline \multirow[t]{6}{*}{ EGB } & NNE & $1.57,1.15$ & $1.53,0.74$ & $0.75,0.47$ & $0.14,0.05$ & $0.12,0.05$ & $0.05,0.04$ & $0.08,0.06$ & $0.99,0.46$ \\
\hline & NNW & $1.37,1.11$ & $1.84,0.90$ & $0.77,0.47$ & $0.32,0.08$ & $0.24,0.07$ & $0.04,0.04$ & $0.09,0.07$ & $1.04,0.54$ \\
\hline & SSE & $5.31,3.42$ & $2.74,1.86$ & $2.26,1.67$ & $0.12,0.04$ & $0.10,0.05$ & $0.08,0.07$ & $0.10,0.06$ & $1.08,0.52$ \\
\hline & WNW & $1.97,1.49$ & $2.66,1.34$ & $1.22,0.77$ & $0.19,0.06$ & $0.14,0.05$ & $0.05,0.04$ & $0.08,0.06$ & $0.78,0.42$ \\
\hline & WNW:L & $3.52,2.30$ & $3.12,2.00$ & $1.76,1.27$ & $0.19,0.06$ & $0.15,0.05$ & $0.07,0.06$ & $0.10,0.07$ & $1.16,0.57$ \\
\hline & WSW & $4.91,3.60$ & $3.45,2.05$ & $2.37,1.83$ & $0.12,0.05$ & $0.10,0.05$ & $0.09,0.08$ & $0.10,0.06$ & $0.87,0.48$ \\
\hline \multirow[t]{6}{*}{ FRE } & ENE & $1.40,1.05$ & $0.73,0.39$ & $0.61,0.46$ & $0.06,0.02$ & $0.10,0.04$ & $0.04,0.04$ & $0.03,0.02$ & $0.22,0.13$ \\
\hline & NNW & $1.17,0.89$ & $0.93,0.65$ & $0.55,0.39$ & $0.24,0.05$ & $0.22,0.09$ & $0.04,0.04$ & $0.04,0.04$ & $0.41,0.30$ \\
\hline & NNW:L & $1.39,1.07$ & $0.97,0.64$ & $0.66,0.50$ & $0.15,0.03$ & $0.15,0.06$ & $0.05,0.04$ & $0.04,0.03$ & $0.35,0.28$ \\
\hline & SSW & $3.51,2.37$ & $1.11,0.64$ & $1.45,1.02$ & $0.09,0.02$ & $0.12,0.04$ & $0.06,0.06$ & $0.03,0.03$ & $0.27,0.18$ \\
\hline & WNW & $2.11,1.51$ & $1.35,0.81$ & $1.02,0.76$ & $0.14,0.03$ & $0.14,0.04$ & $0.05,0.05$ & $0.04,0.03$ & $0.35,0.25$ \\
\hline & WSW & $4.12,2.91$ & $1.84,0.99$ & $1.87,1.48$ & $0.09,0.03$ & $0.11,0.05$ & $0.08,0.07$ & $0.04,0.03$ & $0.37,0.25$ \\
\hline \multirow[t]{6}{*}{ KEJ } & ESE & $1.33,1.00$ & $0.30,0.12$ & $0.26,0.15$ & $0.40,0.08$ & $0.39,0.19$ & $0.04,0.03$ & $0.05,0.03$ & $0.05,0.02$ \\
\hline & NNE & $0.82,0.73$ & $0.24,0.19$ & $0.15,0.11$ & $0.42,0.26$ & $0.40,0.31$ & $0.03,0.02$ & $0.05,0.04$ & $0.04,0.03$ \\
\hline & NNW & $1.16,0.82$ & $0.36,0.20$ & $0.25,0.15$ & $0.44,0.28$ & $0.45,0.34$ & $0.04,0.03$ & $0.06,0.04$ & $0.05,0.04$ \\
\hline & SSW & $2.68,1.60$ & $0.27,0.12$ & $0.51,0.31$ & $0.31,0.04$ & $0.40,0.22$ & $0.06,0.04$ & $0.05,0.03$ & $0.04,0.03$ \\
\hline & WNW & $2.86,1.92$ & $0.54,0.28$ & $0.69,0.47$ & $0.32,0.09$ & $0.47,0.33$ & $0.06,0.05$ & $0.06,0.04$ & $0.08,0.04$ \\
\hline & WNW:L & $1.98,1.27$ & $0.29,0.18$ & $0.42,0.25$ & $0.28,0.07$ & $0.36,0.26$ & $0.05,0.03$ & $0.05,0.03$ & $0.05,0.03$ \\
\hline \multirow[t]{6}{*}{ LED } & ESE & $1.24,0.89$ & $0.15,0.06$ & $0.34,0.21$ & $0.02,0.01$ & $0.06,0.02$ & $0.03,0.03$ & $0.01,0.01$ & $0.05,0.02$ \\
\hline & NNE & $0.58,0.44$ & $0.16,0.09$ & $0.15,0.08$ & $0.04,0.01$ & $0.07,0.05$ & $0.03,0.02$ & $0.01,0.01$ & $0.05,0.02$ \\
\hline & NNW & $0.68,0.50$ & $0.22,0.10$ & $0.18,0.09$ & $0.07,0.01$ & $0.10,0.05$ & $0.02,0.02$ & $0.02,0.01$ & $0.05,0.03$ \\
\hline & WNW & $1.27,0.94$ & $0.40,0.14$ & $0.44,0.23$ & $0.04,0.01$ & $0.09,0.03$ & $0.04,0.03$ & $0.02,0.01$ & $0.06,0.03$ \\
\hline & WNW:L & $1.30,0.84$ & $0.26,0.11$ & $0.40,0.21$ & $0.04,0.01$ & $0.07,0.03$ & $0.04,0.03$ & $0.02,0.01$ & $0.07,0.03$ \\
\hline & WSW & $3.11,1.98$ & $0.46,0.14$ & $1.01,0.68$ & $0.02,0.01$ & $0.06,0.03$ & $0.06,0.05$ & $0.02,0.01$ & $0.09,0.04$ \\
\hline \multirow[t]{6}{*}{ SPR } & ESE & $3.67,2.03$ & $0.38,0.15$ & $1.12,0.65$ & $0.01,0.01$ & $0.04,0.02$ & $0.05,0.04$ & $0.02,0.01$ & $0.14,0.07$ \\
\hline & NNE & $0.99,0.71$ & $0.24,0.09$ & $0.29,0.16$ & $0.02,0.01$ & $0.05,0.02$ & $0.03,0.02$ & $0.02,0.01$ & $0.08,0.03$ \\
\hline & NNW & $0.80,0.70$ & $0.39,0.13$ & $0.27,0.15$ & $0.06,0.01$ & $0.08,0.03$ & $0.03,0.02$ & $0.02,0.01$ & $0.08,0.04$ \\
\hline & WNW & $1.29,0.89$ & $0.63,0.15$ & $0.52,0.25$ & $0.03,0.00$ & $0.05,0.02$ & $0.03,0.03$ & $0.02,0.01$ & $0.11,0.05$ \\
\hline & WNW:L & $2.24,1.37$ & $0.60,0.18$ & $0.78,0.41$ & $0.02,0.01$ & $0.05,0.02$ & $0.04,0.03$ & $0.03,0.02$ & $0.14,0.06$ \\
\hline & WSW & $4.42,2.74$ & $0.92,0.32$ & $1.59,1.06$ & $0.02,0.01$ & $0.04,0.02$ & $0.07,0.06$ & $0.04,0.02$ & $0.19,0.09$ \\
\hline
\end{tabular}


Table 4. Campaign-average species mass concentrations $\left(C, \mu \mathrm{g} \mathrm{m}^{-3}\right)$, fine fraction $\left(\mathrm{PM}_{2.5}\right)$ species concentrations $\left(C{ }_{f}, \mu \mathrm{g} \mathrm{m}{ }^{-3}\right)$ and percentage of mass in the $\mathrm{PM}_{2.5}$ fine fraction $\left(P_{f}, \%\right)$ for $\mathrm{SO}_{4}^{2-}, \mathrm{NO}_{3}^{-}, \mathrm{NH}_{4}^{+}, \mathrm{Cl}^{-}, \mathrm{Na}^{+}, \mathrm{K}^{+}, \mathrm{Mg}^{2+}$ and $\mathrm{Ca}^{2+}$. Standard deviations are shown after the \pm . The mean value of 0 represents a value $<0.005$.

\begin{tabular}{|c|c|c|c|c|c|c|c|c|c|c|c|c|c|c|}
\hline & FRS1 & FRS2 & EGB1 & KEJ1 & KEJ2 & ALG1 & ALG2 & LED1 & LED2 & CHA1 & CHA2 & SPR1 & SPR2 & BRL1 \\
\hline $\mathrm{C}\left(\mathrm{SO}_{4}^{2-}\right)$ & $2.46 \pm 2.35$ & $2.05 \pm 1.88$ & $3.22 \pm 1.87$ & $3.41 \pm 4.4$ & $1.09 \pm 1.2$ & $3.98 \pm 5.23$ & $2.91 \pm 4.37$ & $1.46 \pm 3.76$ & $0.65 \pm 0.79$ & $1.63 \pm 1.69$ & $2.44 \pm 1.62$ & $4.45 \pm 5.84$ & $0.88 \pm 0.38$ & $1.16 \pm 0.63$ \\
\hline $\mathrm{C}_{f}\left(\mathrm{SO}_{4}^{2-}\right)$ & $2.34 \pm 2.31$ & $2 \pm 1.84$ & $3.07 \pm 1.74$ & $3.26 \pm 4.19$ & $0.86 \pm 0.74$ & $3.64 \pm 4.42$ & $2.85 \pm 4.31$ & $1.41 \pm 3.68$ & $0.61 \pm 0.76$ & $1.55 \pm 1.62$ & $2.32 \pm 1.54$ & $4.32 \pm 5.66$ & $0.82 \pm 0.35$ & $1.04 \pm 0.58$ \\
\hline $\mathrm{P}_{f}\left(\mathrm{SO}_{4}^{2-}\right)$ & 95 & 98 & 96 & 96 & 79 & 91 & 98 & 97 & 95 & 95 & 95 & 97 & 93 & 90 \\
\hline $\mathrm{C}\left(\mathrm{NO}_{3}^{-}\right)$ & $1.77 \pm 2.2$ & $0.13 \pm 0.18$ & $3.13 \pm 2.44$ & $0.55 \pm 0.63$ & $0.34 \pm 0.65$ & $3.62 \pm 3.31$ & $0.29 \pm 0.4$ & $0.09 \pm 0.17$ & $0.28 \pm 0.67$ & $0.87 \pm 2.09$ & $0.16 \pm 0.09$ & $0.33 \pm 0.46$ & $0.33 \pm 0.4$ & $2.36 \pm 1.9$ \\
\hline$C_{f}\left(\mathrm{NO}_{3}^{-}\right)$ & $1.52 \pm 1.99$ & $0.05 \pm 0.06$ & $2.8 \pm 2.22$ & $0.17 \pm 0.18$ & $0.09 \pm 0.12$ & $2.83 \pm 2.42$ & $0.07 \pm 0.08$ & $0.02 \pm 0.02$ & $0.23 \pm 0.55$ & $0.73 \pm 1.83$ & $0.05 \pm 0.04$ & $0.11 \pm 0.29$ & $0.26 \pm 0.36$ & $2.07 \pm 1.73$ \\
\hline$P_{f}\left(\mathrm{NO}_{3}^{-}\right)$ & 86 & 37 & 90 & 31 & 25 & 78 & 24 & 23 & 83 & 84 & 28 & 33 & 79 & 88 \\
\hline$C\left(\mathrm{NH}_{4}^{+}\right)$ & $1.37 \pm 1.35$ & $0.77 \pm 0.73$ & $2 \pm 1.24$ & $1.05 \pm 1.22$ & $0.25 \pm 0.38$ & $1.94 \pm 2.54$ & $1.08 \pm 1.59$ & $0.41 \pm 1.06$ & $0.24 \pm 0.45$ & $0.67 \pm 1.06$ & $0.78 \pm 0.54$ & $1.42 \pm 1.89$ & $0.31 \pm 0.23$ & $1.05 \pm 0.63$ \\
\hline$C_{f}\left(\mathrm{NH}_{4}^{+}\right)$ & $1.32 \pm 1.32$ & $0.76 \pm 0.73$ & $1.96 \pm 1.2$ & $1.03 \pm 1.19$ & $0.2 \pm 0.24$ & $1.8 \pm 2.1$ & $1.06 \pm 1.57$ & $0.41 \pm 1.04$ & $0.23 \pm 0.43$ & $0.65 \pm 1.04$ & $0.76 \pm 0.53$ & $1.39 \pm 1.84$ & $0.29 \pm 0.22$ & $0.99 \pm 0.59$ \\
\hline$P_{f}\left(\mathrm{NH}_{4}^{+}\right)$ & 97 & 99 & 98 & 98 & 80 & 92 & 98 & 98 & 95 & 97 & 97 & 98 & 95 & 94 \\
\hline$C^{\prime}\left(\mathrm{Cl}^{-}\right)^{+}$ & $0.06 \pm 0.06$ & $0.01 \pm 0.01$ & $0.23 \pm 0.11$ & $0.16 \pm 0.24$ & $0.42 \pm 0.44$ & $0.48 \pm 0.39$ & $0.01 \pm 0.01$ & $0.05 \pm 0.06$ & $0.02 \pm 0.03$ & $0.12 \pm 0.17$ & $0.01 \pm 0.02$ & $0.01 \pm 0.01$ & $0.02 \pm 0.02$ & $0.07 \pm 0.05$ \\
\hline$C_{f}\left(\mathrm{Cl}^{-}\right)$ & $0.04 \pm 0.06$ & $0.01 \pm 0$ & $0.1 \pm 0.06$ & $0.02 \pm 0.03$ & $0.04 \pm 0.05$ & $0.22 \pm 0.2$ & $0.01 \pm 0.01$ & $0.02 \pm 0.03$ & $0.01 \pm 0.02$ & $0.04 \pm 0.04$ & $0.01 \pm 0.01$ & $0 \pm 0$ & $0.01 \pm 0.01$ & $0.04 \pm 0.03$ \\
\hline$P_{f}\left(\mathrm{Cl}^{-}\right)$ & 71 & 69 & 42 & 14 & 11 & 45 & 58 & 49 & 64 & 34 & 59 & 53 & 49 & 65 \\
\hline$C\left(\mathrm{Na}^{+}\right)$ & $0.06 \pm 0.04$ & $0.02 \pm 0.01$ & $0.19 \pm 0.07$ & $0.24 \pm 0.33$ & $0.34 \pm 0.44$ & $0.43 \pm 0.29$ & $0.02 \pm 0.02$ & $0.05 \pm 0.04$ & $0.02 \pm 0.02$ & $0.15 \pm 0.16$ & $0.02 \pm 0.02$ & $0.03 \pm 0.02$ & $0.04 \pm 0.01$ & $0.04 \pm 0.02$ \\
\hline$C_{f}\left(\mathrm{Na}^{+}\right)$ & $0.03 \pm 0.02$ & $0.01 \pm 0.01$ & $0.07 \pm 0.03$ & $0.07 \pm 0.09$ & $0.05 \pm 0.07$ & $0.22 \pm 0.16$ & $0.01 \pm 0.01$ & $0.03 \pm 0.03$ & $0.01 \pm 0.01$ & $0.07 \pm 0.06$ & $0.01 \pm 0.01$ & $0.02 \pm 0.01$ & $0.02 \pm 0.01$ & $0.02 \pm 0.01$ \\
\hline$P_{f}\left(\mathrm{Na}^{+}\right)$ & 55 & 67 & 35 & 28 & 16 & 51 & 70 & 64 & 69 & 49 & 53 & 64 & 65 & 55 \\
\hline$C\left(\mathrm{~K}^{+}\right)$ & $0.06 \pm 0.04$ & $0.03 \pm 0.01$ & $0.11 \pm 0.05$ & $0.09 \pm 0.07$ & $0.01 \pm 0.02$ & $0.16 \pm 0.15$ & $0.08 \pm 0.08$ & $0.06 \pm 0.03$ & $0.03 \pm 0.03$ & $0.06 \pm 0.06$ & $0.06 \pm 0.03$ & $0.06 \pm 0.03$ & $0.03 \pm 0.02$ & $0.07 \pm 0.07$ \\
\hline$C_{f}\left(\mathrm{~K}^{+}\right)$ & $0.05 \pm 0.03$ & $0.03 \pm 0.01$ & $0.08 \pm 0.03$ & $0.07 \pm 0.07$ & $0.01 \pm 0.01$ & $0.12 \pm 0.13$ & $0.06 \pm 0.07$ & $0.03 \pm 0.02$ & $0.02 \pm 0.02$ & $0.05 \pm 0.05$ & $0.03 \pm 0.02$ & $0.04 \pm 0.03$ & $0.02 \pm 0.01$ & $0.06 \pm 0.06$ \\
\hline$P_{f}\left(\mathrm{~K}^{+}\right)$ & 82 & 80 & 78 & 83 & 36 & 77 & 72 & 55 & 68 & 88 & 50 & 66 & 77 & 81 \\
\hline$C\left(\mathrm{Mg}^{2+}\right)$ & $0.01 \pm 0.01$ & $0 \pm 0.01$ & $0.02 \pm 0.01$ & $0.05 \pm 0.05$ & $0.04 \pm 0.05$ & $0.12 \pm 0.07$ & $0.07 \pm 0.22$ & $0.01 \pm 0.01$ & $0 \pm 0$ & $0.02 \pm 0.03$ & $0.02 \pm 0.01$ & $0.02 \pm 0.01$ & $0.01 \pm 0$ & $0.05 \pm 0.04$ \\
\hline$C_{f}\left(\mathrm{Mg}^{2+}\right)$ & $0 \pm 0$ & $0 \pm 0$ & $0 \pm 0$ & $0.01 \pm 0.01$ & $0.01 \pm 0.01$ & $0.06 \pm 0.05$ & $0.02 \pm 0.09$ & $0 \pm 0$ & $0 \pm 0$ & $0.01 \pm 0.01$ & $0.01 \pm 0$ & $0 \pm 0$ & $0 \pm 0$ & $0.01 \pm 0.01$ \\
\hline$P_{f}\left(\mathrm{Mg}^{2+}\right)$ & 7 & 12 & 23 & 30 & 19 & 45 & 30 & 33 & 51 & 52 & 31 & 21 & 53 & 18 \\
\hline$C\left(\mathrm{Ca}^{2+}\right)$ & $0.14 \pm 0.2$ & $0.14 \pm 0.14$ & $0.23 \pm 0.14$ & $0.12 \pm 0.12$ & $0.02 \pm 0.02$ & $0.41 \pm 0.26$ & $0.2 \pm 0.26$ & $0.05 \pm 0.07$ & $0.02 \pm 0.04$ & $0.09 \pm 0.25$ & $0.1 \pm 0.09$ & $0.16 \pm 0.12$ & $0.04 \pm 0.02$ & $0.21 \pm 0.14$ \\
\hline$C_{f}\left(\mathrm{Ca}^{2+}\right)$ & $0.02 \pm 0.02$ & $0.03 \pm 0.03$ & $0.08 \pm 0.06$ & $0.04 \pm 0.04$ & $0.01 \pm 0.02$ & $0.12 \pm 0.12$ & $0.05 \pm 0.09$ & $0.02 \pm 0.02$ & $0.01 \pm 0$ & $0.03 \pm 0.07$ & $0.03 \pm 0.06$ & $0.03 \pm 0.03$ & $0.02 \pm 0.01$ & $0.03 \pm 0.02$ \\
\hline$P_{f}\left(\mathrm{Ca}^{2+}\right)$ & 13 & 21 & 35 & 34 & 44 & 29 & 26 & 30 & 22 & 37 & 29 & 21 & 41 & 17 \\
\hline
\end{tabular}

Table 5. Mass median aerodynamic diameter (MMAD) (in $\mu \mathrm{m}$ ) and geometric standard deviation (GSD) (separated by “;”) for total, fine $(F)$ and coarse $(C)$ particles for 8 species measured.

\begin{tabular}{|c|c|c|c|c|c|c|c|c|c|c|c|c|c|c|}
\hline & FRS1 & FRS2 & EGB1 & KEJ1 & KEJ2 & ALG1 & ALG2 & LED1 & LED2 & CHA1 & CHA2 & SPR1 & SPR2 & BRL1 \\
\hline $\mathrm{SO}_{4}^{2-}$ & $0.45 ; 2.47$ & $0.28 ; 2.69$ & $0.44 ; 2.4$ & $0.38 ; 2.65$ & $0.67 ; 3.74$ & $0.5 ; 2.7$ & $0.29 ; 2.61$ & $0.44 ; 2.28$ & $0.47 ; 2.37$ & $0.42 ; 2.59$ & $0.42 ; 2.7$ & $0.46 ; 2.39$ & $0.56 ; 2.56$ & $0.43 ; 2.8$ \\
\hline$F\left(\mathrm{SO}_{4}^{2-}\right)$ & $0.4 ; 2.1$ & $0.26 ; 2.42$ & $0.4 ; 2.13$ & $0.35 ; 2.37$ & $0.4 ; 2.38$ & $0.41 ; 2.17$ & $0.27 ; 2.44$ & $0.42 ; 2.12$ & $0.43 ; 2.1$ & $0.38 ; 2.24$ & $0.38 ; 2.37$ & $0.44 ; 2.2$ & $0.49 ; 2.21$ & $0.37 ; 2.26$ \\
\hline$C\left(\mathrm{SO}_{4}^{2-}\right)$ & $4.4 ; 1.45$ & $4.45 ; 1.47$ & $4.16 ; 1.41$ & $4.14 ; 1.45$ & $4.63 ; 1.47$ & $4.39 ; 1.46$ & $4.25 ; 1.47$ & $4.03 ; 1.46$ & $4.01 ; 1.45$ & $4.28 ; 1.47$ & $4.25 ; 1.48$ & $4.04 ; 1.49$ & $4.11 ; 1.45$ & $4.38 ; 1.45$ \\
\hline $\mathrm{NO}_{3}^{-}$ & $0.63 ; 2.91$ & $2.03 ; 3.96$ & $0.55 ; 2.58$ & $3.15 ; 2.64$ & $3.93 ; 2.99$ & $0.73 ; 3.66$ & $3.76 ; 4.25$ & $3.41 ; 4.03$ & $0.79 ; 3.22$ & $0.63 ; 3.4$ & $2.58 ; 5.22$ & $2.41 ; 5.04$ & $0.98 ; 3.08$ & $0.48 ; 2.92$ \\
\hline$F\left(\mathrm{NO}_{3}^{-}\right)$ & $0.46 ; 2.06$ & & $0.44 ; 1.96$ & $1.09 ; 2.66$ & $1.01 ; 3.3$ & $0.43 ; 2.29$ & & & $0.55 ; 2.28$ & $0.44 ; 2.45$ & & & $0.66 ; 2.24$ & $0.38 ; 2.13$ \\
\hline$C\left(\mathrm{NO}_{3}^{-}\right)$ & $4.37 ; 1.42$ & $4.57 ; 1.41$ & $4.38 ; 1.4$ & $4.58 ; 1.44$ & $4.85 ; 1.46$ & $4.94 ; 1.46$ & $5.43 ; 1.43$ & $5.03 ; 1.41$ & $4.61 ; 1.47$ & $4.54 ; 1.45$ & $5.29 ; 1.43$ & $5.11 ; 1.43$ & $4.44 ; 1.46$ & $4.55 ; 1.46$ \\
\hline $\mathrm{NH}_{4}^{+}$ & $0.43 ; 2.25$ & $0.26 ; 2.58$ & $0.41 ; 2.16$ & $0.35 ; 2.44$ & $0.67 ; 3.69$ & $0.46 ; 2.65$ & $0.28 ; 2.67$ & $0.42 ; 2.17$ & $0.5 ; 2.3$ & $0.39 ; 2.47$ & $0.38 ; 2.5$ & $0.45 ; 2.33$ & $0.53 ; 2.52$ & $0.39 ; 2.43$ \\
\hline$F\left(\stackrel{+}{\mathrm{NH}_{4}^{+}}\right)$ & $0.4 ; 2.04$ & $0.25 ; 2.42$ & $0.4 ; 2.05$ & $0.34 ; 2.34$ & $0.42 ; 2.42$ & $0.39 ; 2.17$ & $0.26 ; 2.48$ & $0.4 ; 2.05$ & $0.47 ; 2.09$ & $0.36 ; 2.26$ & $0.36 ; 2.3$ & $0.43 ; 2.21$ & $0.48 ; 2.26$ & $0.35 ; 2.14$ \\
\hline$C\left(\mathrm{NH}_{4}^{+}\right)$ & $4.21 ; 1.43$ & $4.62 ; 1.48$ & $3.63 ; 1.29$ & $4.04 ; 1.48$ & $4.6 ; 1.48$ & $4.51 ; 1.44$ & $4.45 ; 1.51$ & $4.12 ; 1.46$ & $3.88 ; 1.44$ & $4.29 ; 1.48$ & $4.6 ; 1.52$ & $3.76 ; 1.48$ & $3.93 ; 1.43$ & $4.18 ; 1.43$ \\
\hline $\mathrm{Cl}^{-}{ }^{+}$ & & & & $4.38 ; 1.88$ & $5.64 ; 2.07$ & $1.87 ; 5.48$ & & $1.77 ; 4.48$ & & $2.11 ; 6.48$ & & & & $0.86 ; 5.63$ \\
\hline$F\left(\mathrm{Cl}^{-}\right)$ & & & & & & $0.63 ; 5.06$ & & $0.74 ; 4.37$ & & $0.38 ; 6.12$ & & & & $0.38 ; 3.59$ \\
\hline$C\left(\mathrm{Cl}^{-}\right)$ & & & $5.3 ; 1.42$ & $4.89 ; 1.41$ & $5.13 ; 1.43$ & $4.59 ; 1.49$ & & $4.15 ; 1.43$ & & $5.13 ; 1.47$ & & & & $4.84 ; 1.49$ \\
\hline $\mathrm{Na}^{+}$ & & & & $3.49 ; 1.81$ & & $1.75 ; 4.7$ & & $1.28 ; 4.16$ & & $1.79 ; 4.89$ & & & & $1.16 ; 5.01$ \\
\hline$F\left(\mathrm{Na}^{+}\right)$ & & & & & & $0.67 ; 3.79$ & & $0.69 ; 3.61$ & & $0.61 ; 4.06$ & & & & $0.51 ; 3.53$ \\
\hline$C\left(\mathrm{Na}^{+}\right)$ & & & $4.92 ; 1.43$ & $4.49 ; 1.43$ & $4.99 ; 1.44$ & $4.68 ; 1.48$ & & $4.02 ; 1.43$ & & $4.87 ; 1.47$ & & & & $4.59 ; 1.48$ \\
\hline $\mathrm{K}^{+}$ & & & & & & $0.55 ; 5.62$ & & $1.05 ; 6.22$ & & $0.42 ; 4.12$ & $1.38 ; 6.03$ & & & $0.61 ; 3.17$ \\
\hline$F\left(\mathrm{~K}^{+}\right)$ & $0.28 ; 3.2$ & & $0.2 ; 4.6$ & $0.34 ; 2.87$ & & $0.31 ; 3.77$ & $0.26 ; 3.55$ & $0.35 ; 4.19$ & & $0.31 ; 3.01$ & $0.37 ; 3.91$ & & & $0.45 ; 2.35$ \\
\hline$C\left(\mathrm{~K}^{+}\right)$ & & & & & & $4.77 ; 1.5$ & & $4.53 ; 1.44$ & & $4.77 ; 1.52$ & $5.05 ; 1.46$ & & & $4.49 ; 1.45$ \\
\hline $\mathrm{Mg}^{2+}$ & & $3.4 ; 3.76$ & & $3.48 ; 2.31$ & $4.7 ; 2.51$ & $2.56 ; 3.26$ & $2.27 ; 7.78$ & $3.11 ; 3.55$ & & $2.25 ; 2.88$ & $2.91 ; 4.99$ & & & \\
\hline$F\left(\mathrm{Mg}^{2+}\right)$ & & $0.86 ; 3.38$ & & $1.4 ; 2.18$ & $1.23 ; 2.97$ & $1 ; 2.44$ & & $0.92 ; 3.13$ & & $1.07 ; 2.18$ & & & & \\
\hline$C\left(\mathrm{Mg}^{2+}\right)$ & & $4.95 ; 1.47$ & & $4.61 ; 1.45$ & $4.98 ; 1.44$ & $4.93 ; 1.49$ & $5.45 ; 1.45$ & $4.81 ; 1.47$ & & $4.65 ; 1.47$ & $5.18 ; 1.47$ & & & \\
\hline $\mathrm{Ca}^{2+}$ & & & & & & $2.79 ; 5.65$ & $3.43 ; 4.95$ & & & $2.63 ; 4.04$ & $3.17 ; 4.66$ & & & $4.98 ; 2.6$ \\
\hline$F\left(\mathrm{Ca}^{2+}\right)$ & & & & & & $0.36 ; 5.3$ & $0.48 ; 4.73$ & & & $0.7 ; 3.52$ & $0.53 ; 4.16$ & & & $1.21 ; 2.62$ \\
\hline$C\left(\mathrm{Ca}^{2+}\right)$ & $5.27 ; 1.47$ & $5.3 ; 1.46$ & $4.96 ; 1.44$ & $5 ; 1.48$ & & $5.43 ; 1.46$ & $5.4 ; 1.46$ & $5.06 ; 1.46$ & $5.37 ; 1.46$ & $5.1 ; 1.46$ & $5.3 ; 1.46$ & $5.35 ; 1.44$ & & $5.23 ; 1.45$ \\
\hline
\end{tabular}

concentrations (each sample was weighted by its duration) at the six sites that each had two campaigns, (3) the geographical patterns of the campaign-average concentrations, (4) the fine and coarse fractions of the ion mass concentrations, and (5) the characterization of size distributions including the size-distribution profiles and related parameters. Two campaigns made at Algoma are discussed in more detail in Sect. 3.6 considering the strong effects of local sources (road salt) and the unusual seasonal pattern of several observed species (e.g., $\mathrm{SO}_{4}^{2-}, \mathrm{NH}_{4}^{+}$). Size-dependence of particle acidity is explored in Sect. 3.7 based on the chargeequivalent cation/anion ratios at every MOUDI stage.

Fine particles were defined as the particles having an aerodynamic diameter smaller than $2.5 \mu \mathrm{m}\left(\mathrm{PM}_{2.5}\right)$; however, MOUDI stages do not have a size cut at $2.5 \mu \mathrm{m}$. Thus, the measured MOUDI sizes were inverted to give continuous 
size-distribution profiles using a modified Twomey inversion technique (Winklmyr et al., 1990) and kernel functions from Marple et al. (1991), as was done in previous studies (e.g., Li et al., 1998). Fine fractions of the total mass were then obtained by integrating the profiles from $0-2.5 \mu \mathrm{m}$. Within air-quality and climate models where size-resolved aerosols are a concern, a sectional or moment approach is commonly used to describe aerosol size distributions. For the sectional approach, the size-distribution profile presented in Figs. 3 and 4 can be used; for the moment approach, a sum of two or three lognormal distributions is commonly used (e.g., Ackermann et al., 1998). Thus, in the present study, fine and coarse particles are separately fitted into lognormal distributions for potential future applications. The mass median aerodynamic diameter (MMAD) and associated geometric standard deviation (GSD) were calculated for the total mass and for the fine and coarse fractions of the total mass (Table 5). The discussions presented in this section are based on results shown in Tables 4 and 5 and in Figs. 3-6. Information presented in Table 1, Figs. 1 and 2, and Sect. 2.5 is needed to explain the observed phenomenon.

\subsection{Sulphate}

Fine particle $\mathrm{SO}_{4}^{2-}$ is commonly generated by the oxidation of $\mathrm{SO}_{2}$ through a slow gas-phase (homogeneous) oxidation and/or gas/particle phase (heterogeneous) oxidation. The rate of $\mathrm{SO}_{4}^{2-}$ production is expected to be higher in the warm and hot seasons when compared to the cold seasons (see some detailed discussions in Hazi et al., 2003, and references therein). Coarse $\mathrm{SO}_{4}^{2-}$ is produced by reactions of $\mathrm{SO}_{2}$ or sulfuric acid on the wet surface of sea salt or soil particles (Wall et al., 1988).

At Frelighsburg, the average $\mathrm{SO}_{4}^{2-}$ concentration during FRS1 was slightly higher than during FRS2 (Table 4). By looking at the back trajectories shown in Fig. 2, it can be seen that $59 \%$ of the air masses during FRS1 (clusters SSW and WSW) were from the high $\mathrm{SO}_{2}$ emission regions of eastern USA and the industrial areas of southern Ontario and southern Quebec, while only $25 \%$ of the air masses during FRS2 were from these same regions. Thus, despite the slightly colder weather during FRS1, the $\mathrm{SO}_{4}^{2-}$ concentrations were higher because of the more polluted air masses.

At Chalk River, Sprucedale, and Lac Edouard, the hotseason campaigns generally had a higher percentage of polluted air masses (e.g., clusters WSW and ESE clusters were the most polluted and clusters NNW and NNE were the least polluted at all three locations). The campaign-average $\mathrm{SO}_{4}^{2-}$ concentrations were higher during the hot-season campaigns at all three locations due to a combination of the effects of temperature differences and air-mass origins. The campaignaverage $\mathrm{SO}_{4}^{2-}$ concentration was $50 \%$ higher in the hot season compared to the cold season at Chalk River, $>100 \%$ higher at Lac Edouard and $~ 400 \%$ higher at Sprucedale.
At Kejimkujik, the campaign-average $\mathrm{SO}_{4}^{2-}$ concentration was three times higher during the hot-season campaign (KEJ1) compared to the warm-season campaign (KEJ2). This was likely due to a combination of the effects of temperature differences and different air-mass origins. During KEJ1, 28\% of the air masses were from the high emission region (cluster WNW) and 13\% were from the Atlantic Ocean (clusters SSW and ESE) while during KEJ2, these numbers were about the reverse, i.e., $14 \%$ and $25 \%$, respectively. Because of the differences in the air-mass origins during these two campaigns, the concentrations of coarse $\mathrm{SO}_{4}^{2-}$ during KEJ1 were slightly lower than during $\mathrm{KEJ} 2\left(0.15\right.$ vs. $0.23 \mu \mathrm{g} \mathrm{m}^{-3}$ ) while concentrations of fine $\mathrm{SO}_{4}^{2-}$ during KEJ1 were much higher than during KEJ2 (3.3 vs. $0.9 \mu \mathrm{g} \mathrm{m}^{-3}$ ).

At Algoma, the campaign-average $\mathrm{SO}_{4}^{2-}$ concentration was higher during ALG1 than during ALG2. The air-mass origins shown in Fig. 2 cannot explain this phenomenon since ALG1 had a lower percentage of air masses from clusters SSW and ESE (passing over high emission regions) and a higher percentage of air masses from NNW and NNE (passing over clean regions) when compared to ALG2. The phenomenon is also in contradiction to the theory that $\mathrm{SO}_{4}^{2-}$ production was higher during the hot seasons since ALG1 was a cold-season campaign and ALG2 was a hot-season campaign. The causes of higher campaign-average concentrations during the cold-season campaign at this location will be discussed in Sect. 3.6.

In comparing campaign-average concentrations conducted during the same season, higher $\mathrm{SO}_{4}^{2-}$ concentrations were observed at locations close to high $\mathrm{SO}_{2}$ emission areas and/or with air masses from high emission sources. For example, $\mathrm{SO}_{4}^{2-}$ concentrations were higher at locations close to the industrial areas of southern Ontario and southern Quebec (e.g., Sprucedale, Egbert and Frelighsberg) compared to remote locations (e.g., Lac Edouard). The average $\mathrm{SO}_{4}^{2-}$ concentrations varied by $>5$ times from site to site during any season.

The fine fraction of $\mathrm{SO}_{4}^{2-}$ mass concentrations made up $\geq 95 \%$ of the total $\mathrm{SO}_{4}^{2-}$ mass in ten campaigns (FRS1, FRS2, EGB1, KEJ1, ALG2, LED1, LED2, CHA1, CHA2, SPR1), around $90 \%$ in three campaigns (ALG1, SPR2, BRL1), and 79\% in one campaign at a coastal site (KEJ2) in late fall. Note that another campaign at the same coastal site (KEJ1) showed different concentrations ( $>3$ times higher) and fine/total fractions (96\%) from those of KEJ2. The differences were caused by different air-mass origins as shown in Fig. 2. Since fine $\mathrm{SO}_{4}^{2-}$ particles dominated the total $\mathrm{SO}_{4}^{2-}$ mass concentrations, their geographical distributions and seasonal variations were similar to the total $\mathrm{SO}_{4}^{2-}$ mass concentrations (Table 4). Coarse $\mathrm{SO}_{4}^{2-}$ particle concentrations (total minus fine particles) were generally very low, ranging from $0.03-0.32 \mu \mathrm{g} \mathrm{m}^{-3}$ during the fourteen campaigns. 


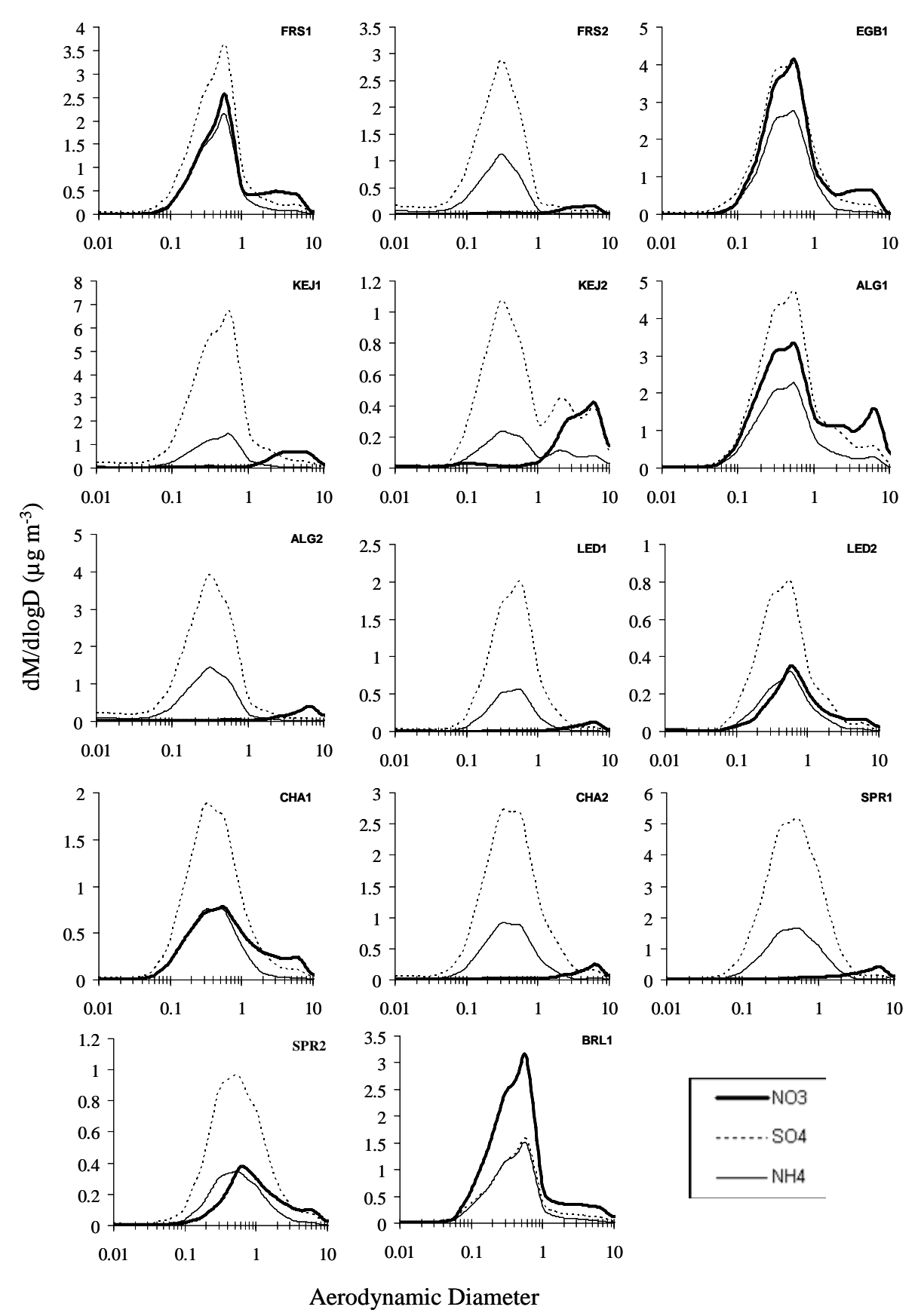

Fig. 3. Average size distributions of $\mathrm{SO}_{4}^{2-}, \mathrm{NH}_{4}^{+}$, and $\mathrm{NO}_{3}^{-}$during 14 field campaigns.

Because of the very small fractions of coarse $\mathrm{SO}_{4}^{2-}$ during most of the campaigns, the $\mathrm{SO}_{4}^{2-}$ size distribution was dominated by a single mode peaking at $0.3-0.6 \mu \mathrm{m}$ (Fig. 3). A campaign-average trimodal size distribution was apparent at $\mathrm{KEJ} 2$ due to the non-negligible coarse $\mathrm{SO}_{4}^{2-}$ fraction. A very small second peak in the coarse particle size range of 2$5 \mu \mathrm{m}$ was also observed in several campaigns (e.g., ALG1, $\mathrm{SPR} 2$ ). In general, the $\mathrm{SO}_{4}^{2-}$ size distribution profiles were similar at different locations and during different seasons; however, there was a tendency for $\mathrm{SO}_{4}^{2-}$ concentrations to peak at slightly larger sizes in the cold seasons $(0.5-0.6 \mu \mathrm{m})$ compared to the hot seasons $(0.3-0.4 \mu \mathrm{m})$. This seasonal difference was identified to be mainly caused by differences in relative humidity $(\mathrm{RH})$ since particles can grow faster under high humidity conditions. The hourly average RH values (Table 1) were generally much higher in the cold seasons compared to the warm/hot seasons, consistent with the larger MMAD values for fine $\mathrm{SO}_{4}^{2-}$ (Table 5). At some locations, 
when the RH seasonal variations were small, the MMAD seasonal variations were also small (e.g., little difference at CHA and very small differences at LED for both RH and MMAD).

The MMAD for $\mathrm{SO}_{4}^{2-}$ over all campaigns, except KEJ2, ranged from 0.28 to $0.56 \mu \mathrm{m}$ with GSDs around 2.3 to 2.8 (Table 5). The KEJ2 campaign exhibited a higher MMAD and GSD due to the contribution of sea salt sulphate particles. By fitting fine and coarse $\mathrm{SO}_{4}^{2-}$ particles separately into lognormal distributions, the MMAD ranged from 0.26 to $0.49 \mu \mathrm{m}$ for fine particles and 4.0 to $4.6 \mu \mathrm{m}$ for coarse particles, with the GSD ranging from 2.1 to 2.4 for fine particles and from 1.4 to 1.5 for coarse particles. Most previous studies showed the MMAD values ranged from $0.3-0.5 \mu \mathrm{m}$ (Milford and Davidson, 1987). Results shown above confirm that the variations in the MMAD and GSD values of $\mathrm{SO}_{4}^{2-}$ were caused by different air-mass origins (which control the emissions of the gaseous precursor) and by meteorological conditions (which control physical and chemical processes that produce $\mathrm{SO}_{4}^{2-}$ and remove it from the atmosphere).

\subsection{Nitrate}

Fine $\mathrm{NO}_{3}^{-}$is produced by the gas-phase reaction of $\mathrm{HNO}_{3}$ with $\mathrm{NH}_{3}$ while coarse $\mathrm{NO}_{3}^{-}$is formed by the heterogeneous reaction of $\mathrm{HNO}_{3}$ gas with sea salt or soil dust particles (Yoshizumi and Hoshi, 1985). The chemistry favours the production of $\mathrm{NH}_{4} \mathrm{NO}_{3}$ at high humidity and low temperature (Allen et al., 1989).

Unlike $\mathrm{SO}_{4}^{2-}$, whose concentrations are usually higher in the hot season, $\mathrm{NO}_{3}^{-}$concentrations are much higher during the cold season due to its favoured low temperature reaction thermodynamics and its volatility in hot weather. This was found to be the case for the campaign-average $\mathrm{NO}_{3}^{-}$concentrations at Frelighsberg, Algoma, Chalk River, and Lac Edouard. It is noted that the $\mathrm{NO}_{3}^{-}$concentrations during FRS1 (November, 2001) were more than ten times higher than during FRS2 (May, 2002) despite the fact that the temperature difference during these two campaigns was small (a few degrees). The very large difference in the campaignaverage $\mathrm{NO}_{3}^{-}$concentrations was caused by a combination of the different air-mass origins and different temperatures since a larger percentage of air-mass trajectories were from the high emission regions and the temperature was lower during FRS1 compared to FRS2. At Sprucedale, no apparent seasonal difference was found, suggesting that the effects of different air-mass origins and the effects of different temperatures during the two campaigns cancelled each other out. At Kejimkujik, the hot-season campaign had a slightly higher average $\mathrm{NO}_{3}^{-}$concentration than the warm-season campaign, but the hot season was determined by coarse particle $\mathrm{NO}_{3}^{-}$, consistent with the considerably higher $\mathrm{Ca}^{2+}$ concentrations and the more frequent trajectories over the high $\mathrm{NO}_{\mathrm{x}}$ emission areas of eastern North America.
Similar to $\mathrm{SO}_{4}^{2-}$, higher $\mathrm{NO}_{3}^{-}$concentrations were found in general at locations close to the high $\mathrm{NO}_{\mathrm{x}}$ and $\mathrm{NH}_{3}$ emission areas and/or with higher frequencies of air masses over the high emission areas. The campaign-average mass concentrations of $\mathrm{NO}_{3}^{-}$ranged from $0.1-3.6 \mu \mathrm{g} \mathrm{m}^{-3}$ depending on location and season (Table 4).

The fraction $\mathrm{NO}_{3}^{-}$mass concentrations in the fine mode were 78-90\% during the seven cold-season campaigns (FRS1, EGB1, ALG1, LED2, CHA1, SPR2, and BRL1) and 23-36\% during the seven warm-season campaigns (FRS2, KEJ1, KEJ2, ALG2, LED1, CHA2, and SPR1). Thus, as expected from thermodynamics, coarse particle $\mathrm{NO}_{3}^{-}$ dominated in the warm seasons while fine particle $\mathrm{NO}_{3}^{-}$ dominated in the cold seasons (except at Kejimkujik and Sprucedale). This also explains the much higher $\mathrm{NO}_{3}^{-}$concentrations during the cold seasons compared to the warm seasons. These results agree with previous studies at different locations (e.g., Kadowaki, 1976; Fisseha et al., 2006). Note that the fine fraction of the total $\mathrm{NO}_{3}^{-}$mass concentrations also depends on the amount of available $\mathrm{NH}_{3}$ as discussed below in Sect. 3.7. The coarse nitrate fractions in the hot seasons discussed above should be treated as an upperend estimation due to the possibility of the loss of fine particle $\mathrm{NH}_{4} \mathrm{NO}_{3}$ collected by MOUDI which could have exaggerated the relative importance of coarse versus fine mode nitrates (Lee et al., 2008).

During the seven cold-season campaigns, the campaignaverage $\mathrm{NO}_{3}^{-}$size distributions showed a bimodal distribution with one peak located in the $0.3-0.6 \mu \mathrm{m}$ range and another in the 4.0-7.0 $\mu \mathrm{m}$ range (Fig. 3). During the seven warm- or hot-season campaigns, $\mathrm{NO}_{3}^{-}$showed only one coarse mode peak at $4.0-7.0 \mu \mathrm{m}$. The size distribution profiles varied significantly with location and season.

The MMAD for fine $\mathrm{NO}_{3}^{-}$ranged from 0.38 to $0.66 \mu \mathrm{m}$ at non-coastal sites, with GSD ranging from 2.0 to 2.3 (Table 5). At the coastal site (KEJ1, KEJ2), the MMAD and GSD were around $1.0 \mu \mathrm{m}$ and 3.0, respectively, larger than at other rural sites. The MMAD and GSD for coarse $\mathrm{NO}_{3}^{-}$ were 4.4-5.4 $\mu \mathrm{m}$ and 1.4-1.47, respectively. The MMAD and GSD values for both fine and coarse $\mathrm{NO}_{3}^{-}$are quite close to previous measurements at different locations (e.g., Ruijgrok et al., 1997).

\subsection{Ammonium}

$\mathrm{NH}_{4}^{+}$is formed from its gaseous precursor $\mathrm{NH}_{3}$ through gasphase and aqueous-phase reactions with acidic species (e.g., $\mathrm{H}_{2} \mathrm{SO}_{4}, \mathrm{HNO}_{3}$, and $\mathrm{HCl}$ ). Among the reaction products, $\left(\mathrm{NH}_{4}\right)_{2} \mathrm{SO}_{4}$ is preferentially formed and the least volatile; $\mathrm{NH}_{4} \mathrm{NO}_{3}$ is relatively volatile and $\mathrm{NH}_{4} \mathrm{Cl}$ is the most volatile. Volatility increases with increasing air temperature and decreasing humidity (Pio et al., 1987; Mozurkewich, 1993). Note that $\mathrm{NH}_{3}$ emissions are considerably higher during the warm/hot seasons than during the cold seasons due to 
increased agricultural activity and temperature-related emissions.

It was noted above that $\mathrm{SO}_{4}^{2-}$ production is higher in the warm/hot seasons. $\mathrm{NH}_{4}^{+}$production is also expected to be higher in the warm/hot seasons due to higher $\mathrm{NH}_{3}$ emissions and the preference of the formation of $\left(\mathrm{NH}_{4}\right)_{2} \mathrm{SO}_{4}$. Thus, the seasonal cycles of $\mathrm{NH}_{4}^{+}$concentrations should be similar to $\mathrm{SO}_{4}^{2-}$ at the same locations. This is consistent with the campaign-average $\mathrm{SO}_{4}^{2-}$ and $\mathrm{NH}_{4}^{+}$concentrations shown in Table 4.

Higher $\mathrm{NH}_{4}^{+}$concentrations were found at locations close to high $\mathrm{NH}_{3}$ emission areas and/or with a higher frequency of air masses that traveled over high $\mathrm{NH}_{3}$ emission areas. For example, very high concentrations of $\mathrm{NH}_{4}^{+}$were observed at Egbert and Bratt's Lake in the cold season. At Egbert, only $6 \%$ of the air masses were from the low $\mathrm{NH}_{3}$ emissions clusters (NNW and NNE). At Bratt's Lake, clusters SSE, WNW L, WNW, NNW all passed over high $\mathrm{NH}_{3}$ areas. Thus, these two campaigns had the highest $\mathrm{NH}_{4}^{+}$concentrations. Both local sources and long-range transport played important roles in the observed high $\mathrm{NH}_{4}^{+}$concentrations. The campaign-average mass concentrations of $\mathrm{NH}_{4}^{+}$ranged from $0.2-2.0 \mu \mathrm{g} \mathrm{m}^{-3}$ across the region.

The fine fraction of $\mathrm{NH}_{4}^{+}$mass concentrations constituted 92-99\% of the total $\mathrm{NH}_{4}^{+}$during thirteen of the fourteen campaigns. The only exception was during KEJ2 when the fine fraction was $80 \%$ of the total. The dominance of the fine $\mathrm{NH}_{4}^{+}$fractions shown in Table 4 and in Fig. 3 suggests that most $\mathrm{NH}_{4}^{+}$was created by homogeneous reactions with a unimodal size distribution peaking at $0.3-0.6 \mu \mathrm{m}$ over most campaigns. A small second mode, with a peak at $6 \mu \mathrm{m}$, was found during ALG1 and a trimodal distribution was found during KEJ2 at a coastal site. Such trimodal distributions have been observed at other coastal locations and are likely caused by a combination of different physical and chemical processes (e.g., aqueous phase chemistry, condensational growth, droplet evaporation, see discussions in Zhuang et al., 1999). The MMAD and GSD for fine $\mathrm{NH}_{4}^{+}$were in the range of $0.25-0.48 \mu \mathrm{m}$ and $2.0-2.5$, respectively, and for coarse $\mathrm{NH}_{4}^{+}$, in the range of 3.6-4.6 $\mu \mathrm{m}$ and 1.3-1.5, respectively. Not surprisingly, these values are very close to those of $\mathrm{SO}_{4}^{2-}$. These two species also had very similar geographical distributions and size distributions.

\subsection{Chloride and sodium}

Most $\mathrm{Cl}^{-}$and $\mathrm{Na}^{+}$measured during the campaigns are thought to be due to sea salt (KEJ1 and KEJ2) and road salt (other campaigns). Note that salt is spread on the roads in the wintertime in many areas of Canada to melt the ice and snow. The campaign-average concentrations for both $\mathrm{Cl}^{-}$and $\mathrm{Na}^{+}$ were very low during most of the campaigns, but as expected, relatively high during KEJ1 and KEJ2 (coastal site). The KEJ2 campaign had much higher concentrations of $\mathrm{Cl}^{-}$and
$\mathrm{Na}^{+}$compared to KEJ1 (0.42 vs. $0.16 \mu \mathrm{g} \mathrm{m}^{-3}$ for $\mathrm{Cl}^{-}$and 0.34 vs. $0.24 \mu \mathrm{g} \mathrm{m}^{-3}$ for $\mathrm{Na}^{+}$). On the other hand, KEJ2 had much lower concentrations of the rest of the species, e.g., the concentrations of $\mathrm{Ca}^{2+}$ and $\mathrm{K}^{+}$during $\mathrm{KEJ} 2$ were only $\sim 15 \%$ of those during KEJ1. The different air-mass origins shown in Fig. 2 certainly played the major role in the concentration differences of all ion species. By comparing the wind speed observed during these two campaigns and the length of 1-day back trajectories (figure not provided), it was found that the trajectory speeds from the Atlantic Ocean were stronger during KEJ2 than during KEJ1. This also caused higher sea salt emissions during KEJ2. During a cold-season campaign at Algoma, very high concentrations of $\mathrm{Cl}^{-}$and $\mathrm{Na}^{+}$were observed, which were identified to be caused by a local source of road salt as will be discussed in Sect. 3.6. Note that the concentration ratio of $\mathrm{Cl}^{-} / \mathrm{Na}^{+}$ranged from 0.3 to 1.2 during thirteen campaigns (1.75 during BRL1), smaller than the seawater ratio of 1.8. This suggested possible $\mathrm{Cl}^{-}$ depletion during most campaigns; and the extent of $\mathrm{Cl}^{-}$depletion should be size-dependent (Yao et al., 2003).

At the coastal site (KEJ1, KEJ2), only around $10 \%$ of the $\mathrm{Cl}^{-}$mass and $15-30 \%$ of the $\mathrm{Na}^{+}$mass were found in the fine fraction. At other locations, the fine fraction of $\mathrm{Cl}^{-}$and $\mathrm{Na}^{+}$mass ranged from $35-70 \%$. This is because of limited sea salt penetration from coastal areas.

Size distributions of $\mathrm{Cl}^{-}$and $\mathrm{Na}^{+}$were very similar during most of the campaigns, except for KEJ1, which exhibited different modes for $\mathrm{Na}^{+}$and $\mathrm{Cl}^{-}$(Fig. 4). For the campaigns with very low concentrations, the size distributions shown in Fig. 4 will be highly uncertain due to large measurement uncertainties. For those campaigns with relatively high concentrations, the size distributions were either unimodal at $\sim 6 \mu \mathrm{m}$ for both $\mathrm{Cl}^{-}$and $\mathrm{Na}^{+}$during EGB1 and KEJ2 or bimodal, with one mode at $2 \mu \mathrm{m}$ and the other at $6 \mu \mathrm{m}$, during ALG1 and CHA1. The size distributions of $\mathrm{Cl}^{-}$and $\mathrm{Na}^{+}$were also unimodal during KEJ1, although at a different mode size. Note that during ALG1, the first mode $(\sim 2 \mu \mathrm{m})$ had higher concentrations than the second mode $(\sim 6 \mu \mathrm{m})$ while during CHA1, the first mode had lower concentrations compared to the second mode.

The MMAD for $\mathrm{Cl}^{-}$and $\mathrm{Na}^{+}$are only presented in Table 5 for those campaigns in which the concentrations were not too low and the data was of relatively good quality. MMAD for both $\mathrm{Cl}^{-}$and $\mathrm{Na}^{+}$were around $4-5 \mu \mathrm{m}$ for the coarse fractions and $0.4-0.7 \mu \mathrm{m}$ for the fine fractions. GSD for the coarse and fine fractions were around 1.5 and 3.5-6, respectively.

\subsection{Potassium, magnesium and calcium}

$\mathrm{Mg}^{2+}$ and $\mathrm{Ca}^{2+}$ are generally from soil particles with a small component from sea salt. $\mathrm{K}^{+}$has additional sources than the other cations, which are mainly soil-derived, that is, biomass burning and vegetation. The sea salt contribution to $\mathrm{K}^{+}$is expected to be very small, except at the coastal 

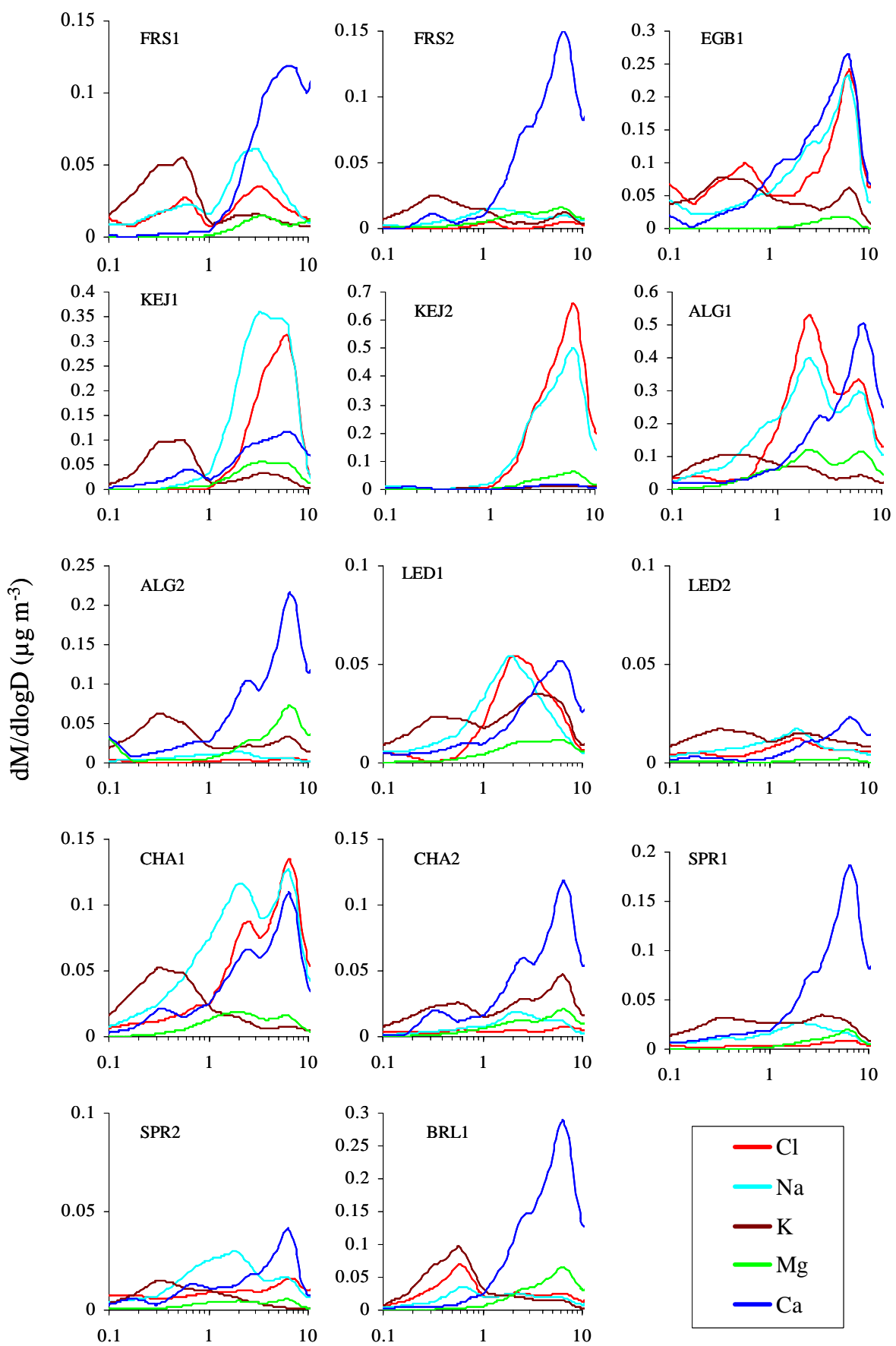

Fig. 4. Average size distributions of $\mathrm{Cl}^{-}, \mathrm{Na}^{+}, \mathrm{K}^{+}, \mathrm{Mg}^{2+}$, and $\mathrm{Ca}^{2+}$ during 14 field campaigns.

site. If assuming all $\mathrm{Na}^{+}$was originated from sea salt particles, the sea salt contribution to $\mathrm{K}^{+}, \mathrm{Mg}^{2+}$, and $\mathrm{Ca}^{2+}$ can be estimated by comparing the observed mass ratio of $\mathrm{K}^{+} / \mathrm{Na}^{+}, \mathrm{Mg}^{2+} / \mathrm{Na}^{+}$, and $\mathrm{Ca}^{2+} / \mathrm{Na}^{+}$with those of seawater, which has a ratio of $0.037,0.12$, and 0.038 , respectively, for $\mathrm{K}^{+} / \mathrm{Na}^{+}, \mathrm{Mg}^{2+} / \mathrm{Na}^{+}$, and $\mathrm{Ca}^{2+} / \mathrm{Na}^{+}$. Such an assumption might not work for winter campaigns conducted at locations close to highways (e.g., ALG1, EGB1) considering the chemical composition of the road salt was not necessarily the same as the sea salt. The mass ratios during the campaign can be inferred from the average mass concentration values shown in Table 4. For cases with extremely low campaignaverage mass concentration values (e.g., $<0.02 \mu \mathrm{g} \mathrm{m}^{-3}$ ), the mass ratio calculated should not be used as the only evidence for estimating the sea salt contributions considering the possible large percentage errors (despite the fact that the absolute error in mass concentration was small). 

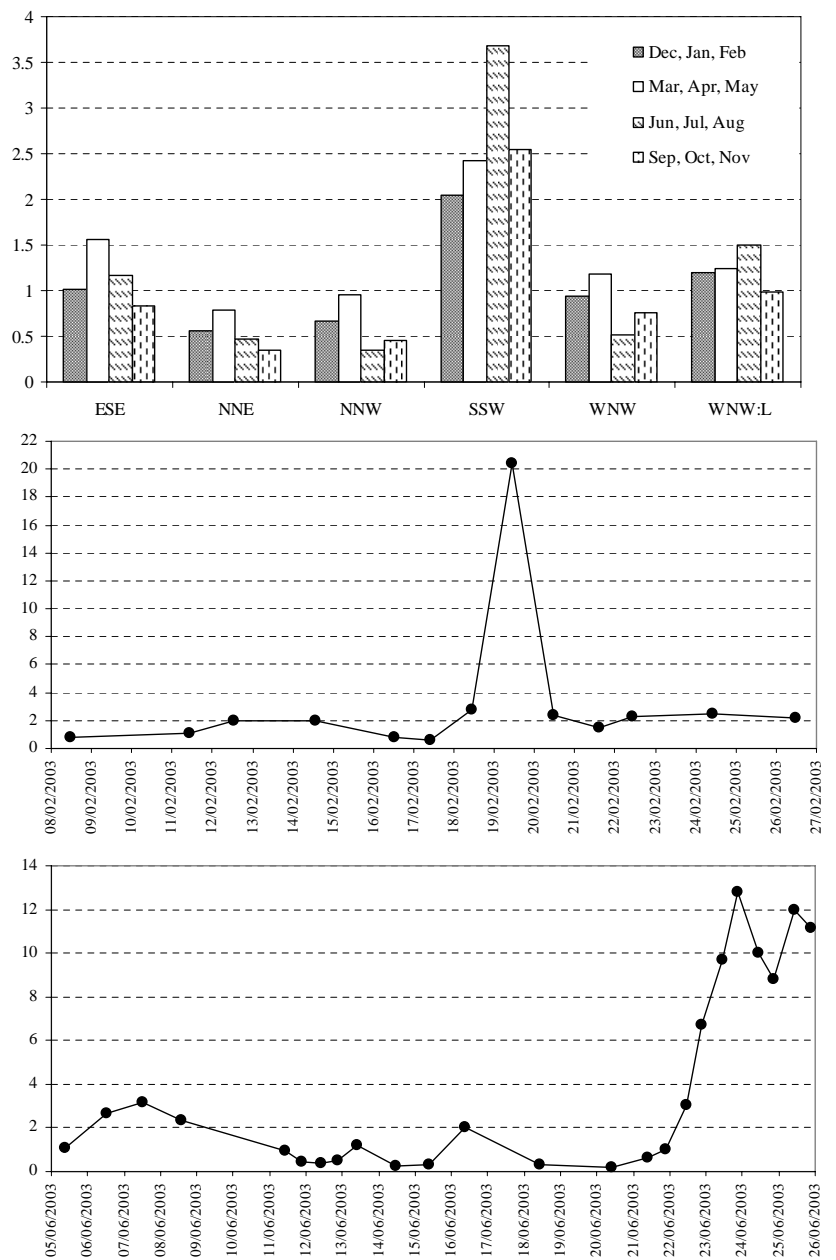

Fig. 5. (a) Median $\mathrm{SO}_{4}^{2-}$ concentration for 6 trajectory clusters from CAPMoN 2001-2005 daily data, (b) and (c) $\mathrm{SO}_{4}^{2-}$ concentrations from individual samples measured during ALG1 and ALG2, respectively.

At the coastal site, the mass ratios of $\mathrm{K}^{+} / \mathrm{Na}^{+}$were found to be 0.03 during one campaign (KEJ2) and 0.37 during another campaign (KEJ1). KEJ2 had the lowest $\mathrm{K}^{+}$concentrations among all of the campaigns, but most of the $\mathrm{K}^{+}$during this campaign was originated from sea salt. On the other hand, only $10 \%$ of $\mathrm{K}^{+}$was identified to be from sea salt during KEJ1. The difference between these two campaigns was caused by different air-mass origins as discussed in Sect. 3.4. The mass ratios of $\mathrm{K}^{+} / \mathrm{Na}^{+}$were found to be in the range of 1.2-4.0 during the rest of the non-winter campaigns. This suggests that sea salt played no significant role $(<3 \%)$ as a source of $\mathrm{K}^{+}$at inland sites.

The mass concentrations of $\mathrm{Mg}^{2+}$ were very low $\left(\leq 0.02 \mu \mathrm{g} \mathrm{m}^{-3}\right.$ ) during nine campaigns. During the two campaigns made at the coastal site, the mass ratios of $\mathrm{Mg}^{2+} / \mathrm{Na}^{+}$were found to be 0.21 (KEJ1) and 0.12 (KEJ2), suggesting that $\sim 50 \%$ of the $\mathrm{Mg}^{2+}$ during KEJ1 and all of the $\mathrm{Mg}^{2+}$ during KEJ2 were from sea salt. The only non-winter inland campaign that had $\mathrm{Mg}^{2+}$ concentrations higher than $0.02 \mu \mathrm{g} \mathrm{m}^{-3}$ is ALG2, which had a mass ratio of $\mathrm{Mg}^{2+} / \mathrm{Na}^{+}$equal to 3.5, suggesting little influence of sea salt. Similarly, $7 \%$ and $60 \%$ of the $\mathrm{Ca}^{2+}$ during KEJ1 and KEJ2, respectively, were from sea salt. For all the rest of the campaigns, sea salt played no significant role in the mass concentration of $\mathrm{Ca}^{2+}$.

Fine particle $\mathrm{K}^{+}$concentrations dominated its total mass concentrations during most of the campaigns. The almost equal amounts of $\mathrm{K}^{+}$in the fine and coarse fractions during KEJ1 and a few other campaigns suggest that emissions from soil dust and emissions from biomass burning and vegetation were equally important. Enhanced $\mathrm{K}^{+}$concentrations in submicron particles were also found in previous studies (Andreae, 1983; Gaudichet et al., 1995; Jaffrezo et al., 1998). The size distribution of $\mathrm{K}^{+}$was bimodal, with one mode peaking at $0.3-0.5 \mu \mathrm{m}$ and the other at $4-6 \mu \mathrm{m}$.

Coarse particle $\mathrm{Mg}^{2+}$ and $\mathrm{Ca}^{2+}$ concentrations dominated their respective total mass concentrations during most of the campaigns. $\mathrm{Mg}^{2+}$ and $\mathrm{Ca}^{2+}$ had bimodal distributions (one at $2.5 \mu \mathrm{m}$ and the other at $6 \mu \mathrm{m}$ ) during several campaigns. In a few campaigns, an extra fine mode was also observed (e.g., $\mathrm{Ca}^{2+}$ in CHA1 and CHA2); however, the concentrations were extremely low and thus, the size distributions shown in Fig. 4 might not represent the real situation. The size distribution of $\mathrm{Mg}^{2+}$ shown for ALG1 seems to be real since it also had two modes similar to the other cation species. The multimodal distributions have also been observed at other locations (e.g., Lin et al., 2007). Such a phenomenon could be caused by a mix of different-aged air masses having different modes, e.g., one air mass having new emissions peaking at sizes of $6 \mu \mathrm{m}$ in diameter and another one having an aged air mass peaking at submicron sizes, the latter could be due to the lowest removal rate by dry deposition and by precipitation scavenging for this particle size range.

The MMAD for fine and coarse $\mathrm{K}^{+}$were $0.4-1.4 \mu \mathrm{m}$ and $5 \mu \mathrm{m}$, respectively. The MMAD for $\mathrm{Mg}^{2+}$ were 0.8 $1.4 \mu \mathrm{m}$ for fine fractions and around $5 \mu \mathrm{m}$ for coarse fractions, and the corresponding GSD were $2-3$ and 5 , respectively. MMAD and GSD for coarse $\mathrm{Ca}^{2+}$ were around $5 \mu \mathrm{m}$ and 1.5 , respectively.

\subsection{Comparison of campaigns ALG1 and ALG2}

Differences between the two campaigns conducted at the same locations were mostly explained by differences in airmass origins (through back trajectory cluster analysis) and seasonal differences in meteorology conditions (e.g., temperature, relative humidity). However, the two campaigns made at Algoma seemed to not be fully explained by the same approach. Further reasoning is needed to explain the extremely high concentrations of $\mathrm{Cl}^{-}$and $\mathrm{Na}^{+}$in the winter and the observed higher $\mathrm{SO}_{4}^{2-}$ concentrations in the winter instead 

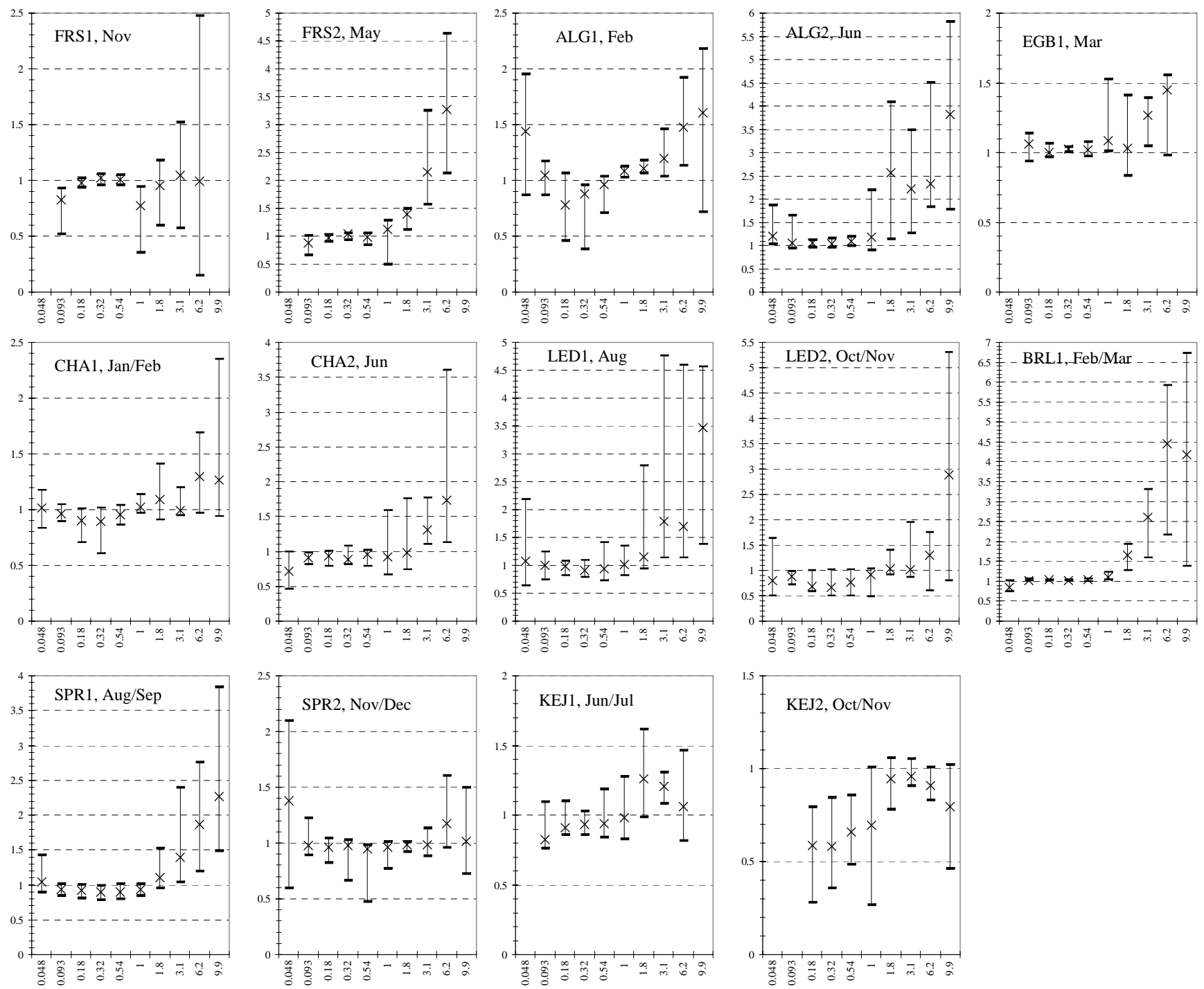

Fig. 6. Statistics of cation-anion ratios at every MOUDI stage during the 14 campaigns. The symbol " $x$ " represents median value and the vertical line represents the range of the central $67 \%$ data.

of in the summer. Thus, individual samples collected during ALG1 and ALG2 and their associated trajectory clusters and local meteorology were studied in detail.

It was found that the majority of the samples from ALG1 had much higher $\mathrm{Cl}^{-}$and $\mathrm{Na}^{+}$concentrations than during ALG2, in particular three samples during ALG1. The TransCanada Highway, a major transport route, is located $12 \mathrm{~km}$ southwest from this site at its closest point. During the winter season, road salt $\left(\mathrm{MgCl}_{2}\right.$ in solution and $\left.\mathrm{NaCl}\right)$ and sand (probably contains $\mathrm{Ca}^{2+}$ and $\mathrm{Mg}^{2+}$ ) was heavily used to help melt the ice and snow on this highway. Winds coming from any direction between the south and northwest (150$315^{\circ}$ ) would have brought the air to this site that passed over the highway. Thus, the much higher $\mathrm{Cl}^{-}$and $\mathrm{Na}^{+}$ concentrations during ALG1 $\left(>0.4 \mu \mathrm{g} \mathrm{m}^{-3}\right)$ compared to
ALG2 $\left(<0.02 \mu \mathrm{g} \mathrm{m}^{-3}\right)$ were thought to be caused by the local source of the road salt. The five-year (2001-2005) CAP$\mathrm{MoN}$ daily concentrations also showed that the average concentrations of $\mathrm{Cl}^{-}$and $\mathrm{Na}^{+}$in the winter (December, January, February) were $\sim 5$ times higher than the rest of the seasons. It also explains why when $\mathrm{Cl}^{-}$and $\mathrm{Na}^{+}$were high, enhanced $\mathrm{Ca}^{2+}$ and $\mathrm{Mg}^{2+}$ were also sometimes detected.

As mentioned in Sect. 3.1, ALG1 had a lower percentage of air masses from clusters SSW and ESE (passing over high emission regions) and a higher percentage of air masses from NNW and NNE (passing over clean regions) when compared to ALG2. The $\mathrm{SO}_{4}^{2-}$ production rate was also expected to be higher during ALG2 (the hot-season campaign). By looking at the median and mean $\mathrm{SO}_{4}^{2-}$ concentrations associated with the different trajectory clusters from 
the five-year (2001-2005) CAPMoN daily data, it was found that the clusters associated with the high emission areas had higher $\mathrm{SO}_{4}^{2-}$ concentrations in the summer than in the winter while the clusters associated with the clean areas had lower $\mathrm{SO}_{4}^{2-}$ concentrations in the summer than in the winter (Fig. 5a, only median values were shown here, mean values have similar seasonal patterns). For example, clusters SSW, ESE, and WNW_L had higher $\mathrm{SO}_{4}^{2-}$ concentrations in the summer while clusters NNE, NNW, and WNW had lower $\mathrm{SO}_{4}^{2-}$ concentrations in the summer compared to the winter. The higher $\mathrm{SO}_{4}^{2-}$ concentrations in the summer from the polluted-clusters were caused by the higher $\mathrm{SO}_{4}^{2-}$ production rate. One cause of the lower $\mathrm{SO}_{4}^{2-}$ concentrations in the summer compared to the winter from the clean-clusters was probably due to the fast removal rate (dry and wet deposition). However, further studies are needed to identify all the possible causes. It is noted that many samples during ALG1 associated with clean-clusters had a concentration of $\sim 2 \mu \mathrm{g} \mathrm{m}^{-3}$ while many samples during ALG2 had a concentration of $\leq 0.5 \mu \mathrm{g} \mathrm{m}^{-3}$ (Fig. 5b and c). This partially explains the higher $\mathrm{SO}_{4}^{2-}$ concentrations during ALG1 than during ALG2.

Another reason for the higher $\mathrm{SO}_{4}^{2-}$ mass concentrations during ALG1 is that there was one 24-h sample (starting at 11:30 on 19 February) that had a very high concentration $\left(\sim 20 \mu \mathrm{g} \mathrm{m}^{-3}\right)$. The sample was associated with clusters SSW and WNW_L and the extremely low wind speed on 19 February. As noted in Sect. 2.5, the slow-moving air mass passing over the high emission areas causes high pollutant concentrations, which likely explains the very high concentration of the sample collected on 19 February. It is worth mentioning that the very low mixing heights in the winter will likely have also played a role in the high pollutant concentrations associated with the polluted air masses. As a comparison, ALG2 had seven samples during the threeday period (23-25 June) associated with cluster SSW. The concentrations of these seven samples were much higher than the rest of the samples (e.g., $6-12 \mu \mathrm{g} \mathrm{m}^{-3}$ versus $0.5-$ $3 \mu \mathrm{g} \mathrm{m}^{-3}$ ), but substantially lower than the extreme sample collected on 19 February during ALG1. Thus, despite the higher percentage of air masses associated with polluted trajectory clusters during ALG2, the campaign-average concentrations were $30 \%$ lower compared to ALG1. Several other species $\left(\mathrm{NO}_{3}^{-}, \mathrm{NH}_{4}^{+}, \mathrm{K}^{+}\right.$, and $\left.\mathrm{Ca}^{2+}\right)$ had day-to-day variations similar to $\mathrm{SO}_{4}^{2-}$, as shown in Fig. $5 \mathrm{~b}$ and c. Thus, these species also had higher campaign-average concentrations during ALG1 than during ALG2, although the magnitudes of the differences between the two campaigns were different for the different species due to their different chemical mechanisms and emission sources.

\subsection{Size-dependence of particle acidity}

Fine particles are known to be responsible for many adverse health effects for which aerosol acidity seems to play a role (see discussions and references in Haze et al., 2003, and Moya et al., 2003). While it is generally believed that submicron particles are more acidic than supermicron particles, there is no consistent conclusion on the size-dependence of the acidity within the size range of submicron particles (see a detailed discussion and references in Yao et al., 2007). Data collected in the present study can shed some light on how the particle acidity changes with particle size. In this section, the concentration ratios (in charge equivalents) between the sum of the measured cations $\left(\mathrm{NH}_{4}^{+}, \mathrm{K}^{+}, \mathrm{Na}^{+}, \mathrm{Mg}^{2+}\right.$, and $\left.\mathrm{Ca}^{2+}\right)$ and the sum of the measured anions $\left(\mathrm{SO}_{4}^{2-}, \mathrm{NO}_{3}^{-}\right.$, and $\mathrm{Cl}^{-}$) were calculated separately for every sample and each MOUDI stage, similar to the approach described in Kerminen et al. (2001). The statistics of the cation/anion ratios (median and the central 67\% values) are shown in Fig. 6 and are discussed in detail below. Note that the data from the first and the last stage were not included considering their relatively poor data quality (due to the very low ion concentrations) compared to the other stages.

Supermicron particles were found to be weakly acidic during two campaigns (FRS1 and KEJ2), close to neutral during one campaign (SPR2) and alkaline during the rest of the campaigns. Kerminen et al. (2001) suggested that carbonate particles associated with mineral dust probably played a major role in the alkalinity of supermicron particles. The depletion of $\mathrm{Cl}^{-}$might have also played an important role considering the much smaller $\mathrm{Cl}^{-} / \mathrm{Na}^{+}$ratio compared to seawater (see the discussion in Sect. 3.4). The acidic supermicron particles observed during KEJ2 were certainly caused by the very low concentrations of the dust-originating ion species $\left(\mathrm{Ca}^{2+}\right.$, $\mathrm{Mg}^{2+}, \mathrm{K}^{+}$), as seen in Table 4, due to the large percentage of ocean air masses reaching this location (Fig. 2).

At the three sites having the highest $\mathrm{NH}_{3}$ emissions (see Sect. 2.5), the submicron particles were basically neutral, even during the cold seasons, i.e., the four campaigns (FRS1, FRS2, EGB1, and BRL1) conducted at the three sites. At the rural locations, where $\mathrm{NH}_{3}$ emissions were not so high, the submicron particles were neutral or close to neutral during the hot seasons (e.g., ALG2, CHA2, LED1, SPR1), but were acidic during the cold seasons (e.g., ALG1, CHA1, LED2, SPR2). This is because more $\mathrm{NH}_{3}$ was available in the warm/hot seasons than in the cold seasons to neutralize the acidic species. At the coastal site, where $\mathrm{NH}_{3}$ emissions were extremely low, the submicron particles were acidic. The acidity was stronger during KEJ2 than during KEJ1 due to the larger percentage of air masses from the ocean. The strong acidity of the submicron particles observed at KEJ is consistent with previously reported high incidences of acidic particles in Atlantic Canada (Brook et al., 1997).

The accumulation mode particles $(0.1-1 \mu \mathrm{m})$ were more acidic than the nucleation mode particles $(<0.1 \mu \mathrm{m})$ during 
the campaigns that had strong acidic submicron particles (see campaigns ALG1, CHA1, LED2, KEJ1, and KEJ2). The strongest acidic particles were from the MOUDI stages 0.18, 0.32 , and $0.54 \mu \mathrm{m}$. Such a phenomenon was consistent with what was observed by Kerminen et al. (2001) at remote sites and the calculated in situ $\mathrm{pH}$ at an urban/coastal site (Yao et al., 2007). Kerminen et al. (2001) suggested that the phenomenon was likely caused by in-cloud sulphate production, which made the smallest accumulation mode particles more acidic than the non-activated nucleation mode particles and Yao et al. (2007) suggested that it was caused by the faster neutralization rate of the smaller particles. On the other hand, the present study showed no apparent dependence of particle acidity on particle size during the campaigns that only had weakly acidic particles (e.g., campaigns CHA2, LED1, SPR1, SPR2).

It is noted that for the cases where there was enough $\mathrm{NH}_{3}$ to neutralize the acidic species, the accumulation mode particles were all close to neutral with no size-dependence; however, the nucleation mode particles were alkaline during some of the campaigns (e.g., campaigns ALG2, EGB1) and were acidic during a few of the other campaigns (campaigns FRS1, FRS2, BRL1). Clearly, the dependence of particle acidity on particle size from the nucleation mode to the accumulation mode was not consistent from site to site or from season to season. The inconsistence of the dependence of particle acidity on particle size should have been caused by the different emission sources of all the related species and the different meteorological conditions during the different campaigns. This might explain the controversial phenomenon found in previous studies as discussed in Yao et al. (2007).

\section{Conclusions and discussions}

Fourteen short-term field campaigns were conducted at eight selected Canadian rural sites to measure size distributions of $\mathrm{SO}_{4}^{2-}, \mathrm{NO}_{3}^{-}, \mathrm{NH}_{4}^{+}, \mathrm{Cl}^{-}, \mathrm{K}^{+}, \mathrm{Na}^{+}, \mathrm{Mg}^{2+}$, and $\mathrm{Ca}^{2+}$ using a Micro-Orifice Uniform Deposit Impactor. Measurement data show that $\mathrm{SO}_{4}^{2-}$ and $\mathrm{NH}_{4}^{+}$were predominately in the fine particle mode at most sites. Fine mode $\mathrm{NO}_{3}^{-}$dominated its concentrations during the cold seasons while coarse mode dominated during the warm/hot seasons. Fine mode $\mathrm{K}^{+}$and coarse mode $\mathrm{Mg}^{2+}$ and $\mathrm{Ca}^{2+}$ dominated their respective mass concentrations. Coarse particles of $\mathrm{Cl}^{-}$and $\mathrm{Na}^{+}$dominated their total concentrations at a coastal site, although substantial amounts of fine particles were also observed at locations far from the seashore. A bimodal distribution of $\mathrm{SO}_{4}^{2-}$ and $\mathrm{NH}_{4}^{+}$was found during one campaign, a trimodal distribution during another campaign made at a coastal site, and a unimodal distribution during the rest of the campaigns. A bimodal distribution, one fine mode and one coarse mode, of $\mathrm{NO}_{3}^{-}$was found during the cold-season campaigns and a unimodal distribution was found in the coarse mode during the rest of the campaigns. A unimodal size distribution in the coarse mode was found for $\mathrm{Cl}^{-}, \mathrm{Na}^{+}, \mathrm{Mg}^{2+}$, and $\mathrm{Ca}^{2+}$ during half of the campaigns and a bimodal distribution, both in the coarse mode, was found during the rest of the campaigns. A bimodal distribution, one in the fine mode and one in the coarse mode, of $\mathrm{K}^{+}$was observed during most of the campaigns. While emission sources and air mass origins were important to the size distribution of the primary particles, meteorological conditions were found to be more important for the secondary particles. This can be seen from the completely different size distribution profiles of $\mathrm{NO}_{3}^{-}$and the different peak sizes of $\mathrm{SO}_{4}^{2-}$ during the different seasons. In general, the size distribution profiles of the eight inorganic ions presented here agree quite well with previous studies conducted at different locations around the world.

Supermicron particles were generally close to neutral or even alkaline in this region except at the coastal location when substantial ocean air masses were presented. The acidity of the submicron particles ranged from strongly acidic to neutral to slightly alkaline. When submicron particles were in the strongly acidic state, the accumulation mode particles were more acidic than the nucleation mode particles; this was not always the case when the submicron particles were in the states between weakly acidic and weakly alkaline. It is concluded that the acidity of fine particles and the dependence of the particle acidity on the particle size for submicron particles will vary with location and season due to the different emission sources of all involved species and the different meteorological conditions. Further studies are needed to identify the major factors affecting the size-dependent particle acidity.

Daily bulk concentrations of the inorganic species considered in this study have been monitored in eastern Canada at multiple locations for more than two decades so their seasonal and annual variations can be easily identified. However, knowledge on their size distributions, especially their geographical and seasonal patterns, is limited. Results presented in this study have shed some light on this topic and will be useful for improving future air-quality, climate and health studies. Although the selected eight sites for the field campaigns cover both polluted and clean areas of southeastern Canada, they might not represent the far remote areas of northern Canada where few anthropogenic emissions exist. Besides, the land types and climate conditions in the northern region are also quite different from the southern region. It should also be pointed out that at the two polluted sites (EGB and BRL), only one cold-season campaign was conducted, and at the third polluted site (FRS), one campaign was conducted in the cold season and the other in the warm (instead of hot season). Thus, the seasonal variations at these three polluted sites cannot be explored. Nevertheless, the seasonal and geographical features shown in this study should provide some scientific evidence for improving the databases at locations where only limited data are available. 
Acknowledgements. We greatly appreciate CAPMoN lab for analyzing MOUDI data and J. Bottenheim, J. R. Brook, S.-M. Li, P. Makar, L. P. Murphy and S.-H. Park for helpful discussions.

Edited by: C. Chan

\section{References}

Abdalmogith, S. S. and Harrison, R. M.: An analysis of spatial and temporal properties of daily sulphate, nitrate and chloride concentrations at UK urban and rural sites, J. Environ. Monitor., 8, 691-699, 2006.

Abdalmogith, S. S. and Harrison, R. M.: The use of trajectory cluster analysis to examine the long-range transport of secondary inorganic aerosol in the UK, Atmos. Environ., 39, 6686-6695, 2005.

Ackermann, I. J., Hass, H., Memmesheimer, M., Ebel, A., Binkowski, F. S., and Shankar, U.: Modal aerosol dynamics model for Europe: Development and first applications, Atmos. Environ., 32, 2981-2999, 1998.

Allen, A. G., Harrison, R. M., and Erisman, J. W.: Field Measurements of the dissociation of ammonium nitrate and ammonium chloride aerosols, Atmos. Environ., 26, 1591-1599, 1989.

Andreae, M. O.: Soot carbon and excess fine potassium: long-range transport of combustion-derived aerosols, Science, 220, 1148$1151,1983$.

Andreae, M. O. and Crutzen, P. J.: Atmospheric aerosols: Biogeochemical sources and role in atmospheric chemistry, Science, 276(5315), 1052-1058, 1997.

Birmili, W., Wiedensohler, A., Heintzenberg, J., and Lehmann, K.: Atmospheric particle number size distribution in central Europe: Statistical relations to air masses and meteorology, J. Geophys. Res., 106, 32 005-32 018, 2001.

Brook, J. R., Wiebe, A. H., Woodhouse, S. A., Audette, C. V., Dann, T. F., Callaghan, S., Piechowski, M., Dabek-Zlotorzynska, E., and Dloughy, J. F.: Temporal and spatial relationships in fine particle strong acidity, sulphate and PM across multiple Canadian locations, Atmos. Environ., 31, 4223-4236, 1997.

Chen, Y.-Q., Zhang, Y., and Zhang, X.-S.: Size distribution and seasonal variation of ions in aerosol at semi-urban site in Beijing, Acta Ecologica Sinica, 25, 3231-3236, 2005.

Dorling, S. R., Davies, T. D., and Pierce, C. E.: Cluster analysis: a technique for estimating the synoptic meteorological controls on air and precipitation chemistry method and applications, Atmos. Environ., 26, 2575-2581, 1992.

Environment Canada: 2004 Canadian acid deposition science assessment, 2005.

Fisseha, R., Dommen, J., Gutzwiller, L., Weingartner, E., Gysel, M., Emmenegger, C., Kalberer, M., and Baltensperger, U.: Seasonal and diurnal characteristics of water soluble inorganic compounds in the gas and aerosol phase in the Zurich area, Atmos. Chem. Phys., 6, 1895-1904, 2006, http://www.atmos-chem-phys.net/6/1895/2006/.

Gaudichet, A., Echalar, F., Chatenet, B., Quisefit, J. P., Malingre, G., Cachier, H., Buat-Menard, P., Artaxo, P., and Maenhaut, W.: Trace elements in tropical African savanna biomass burning aerosols, J. Atmos. Chem., 22, 19-39, 1995.

Gong, W., Dastoor, A. P., Bouchet, V. S., Gong, S., Makar, P. A., Moran, M. D., Pabla, B., Menard, S., Crevier, L.-P., Cousineau,
S., and Venkatesh, S.: Cloud processing of gases and aerosols in a regional air quality model (AURAMS), Atmos. Res., 82, 248275, 2006.

Hazi, Y., Heikkinen, M. S. A., and Cohen, B. S.: Size distribution of acidic sulfate ions in fine ambient particulate matter and assessment of source region effect, Atmos. Environ., 37, 5403-5413, 2003.

Heintzenberg, J., Covert, D. C., and Van Dingenen, R.: Size distribution and chemical composition of marine aerosols: A compilation and review, Tellus Series B, 52, 1104-1122, 2000.

Hillamo, R., Allegrini, I., Sparapani, R., and Kerminen, V.-M.: Mass size distributions and precursor gas concentrations of major inorganic ions in Antarctic aerosol, Int. J. Environ. Anal. Chem., 71, 353-372, 1998.

IPCC: Climate Change 2007: The Physical Science Basis, in: Contribution of Working Group I to the Fourth Assessment Report of the Intergovernmental Panel on Climate Change, edited by: Solomon, S., Qin, D., Manning, M., Chen, Z., Marquis, M., Averyt, K. B., Tignor, M., and Miller, H. L., Cambridge University Press, Cambridge, United Kingdom and New York, NY, USA, 996 pp., 2007.

Jaffrezo, J.-L., Davidson, C. I., Kuhns, H. D., Bergin, M. H., Hillamo, R., Maenhaut, W., Kahl, J. W., and Harris, J. M.: Biomass burning signatures in the atmosphere of central Greenland, J. Geophy. Res., 103,31 067-31 078, 1998.

Kadowaki, S.: Size distribution and chemical composition of atmospheric particulate nitrate in the Nagoya area, Atmos. Environ., 11, 671-675, 1977.

Kaufman, Y. J., Tanré, D., and Boucher, O.: A satellite view of aerosols in the climate system, Nature, 419(6903), 215-223, 2002.

Kerminen, V.-M., Hillamo, R., Teinilä, K., Pakkanen, T., Allegrini, I., and Sparapani, R.: Ion balances of size-resolved tropospheric aerosol samples: Implications for the acidity and atmospheric processing of aerosols, Atmos. Environ., 35, 5255-5265, 2001.

Krivácsy, Z. and Molnár, A.: Size distribution of ions in atmospheric aerosols, Atmos. Res., 46, 279-291, 1998.

Lee, T., Yu, X.-Y., Ayres, B., Kreidenweis, S. M., Malm, W. C., and Collett Jr., J. L.: Observations of fine and coarse particle nitrate at several rural locations in the United States, Atmos. Environ., 42, 2720-2732, 2008.

Lestari, P., Oskouie, A. K., and Noll, K. E.: Size distribution and dry deposition of particulate mass, sulfate and nitrate in an urban area, Atmos. Environ., 37, 2507-2516, 2003.

Li, S.-M., Strawbridge, K. B., Leaitch, W. R., and Macdonald, A. M.: Aerosol backscattering determined from chemical and physical properties and lidar observations over the east coast of Canada, Geophys. Res. Lett., 25, 1653-1656, 1998.

Lin, C.-C., Chen, S.-J., Huang, K.-L., Lee, W.-J., Lin, W.-Y., Liao, C.-J., Chaung, H.-C., and Chiu, C.-H.: Water-soluble ions in nano/ultrafine/fine/coarse particles collected near a busy road and at a rural site, Environ. Pollut., 145, 562-570, 2007.

Marple, V. A., Rubow, K. L., and Behm, S. M.: A micro orifice uniform deposit impactor (MOUDI): Description, calibration, and use, Aerosol Sci. Technol., 14, 434-446, 1991.

McMurry, P. H.: A review of atmospheric aerosol measurements, Atmos. Environ., 34, 1959-1999, 2000.

McNaulty, S. G., Cohen, E. C., Moore Myers, J. A., Sullivan, T. J., and Li, H.: Estimates of critical acid loads and exceedances 
for forest soils across the conterminous United States, Environ. Pollut., 149, 281-292, 2007.

Milford, J. B. and Davidson, C. I.: The sizes of particulate sulphate and nitrate in the atmosphere, J. Air Pollut. Control Assoc., 37, 125-134, 1987.

Moya, M., Ansari, A. S., and Pandis, S. N.: Partitioning of nitrate and ammonium between the gas and particulate phases during the 1997 IMADA-AVER study in Mexico City, Atmos. Environ., 35, 1791-1804, 2001.

Mozurkewich, M.: The dissociation constant of ammonium nitrate and its dependence on temperature, relative humidity and particle size, Atmos. Environ., 27, 261-270, 1993.

Park, S. S. and Kim, Y. J.: $\mathrm{PM}_{2.5}$ particles and size-segregated ionic species measured during fall season in three urban sites in Korea, Atmos. Environ., 38, 1459-1471, 2004.

Park, S. H., Song, C. B., Kim, M. C., Kwon, S. B., and Lee, K. W.: Study on size distribution of total aerosol and water-soluble ions during an Asian dust storm event at Jeju Island, Korea, Environ. Monitor. Assess., 93, 157-183, 2004.

Parmar, R. S., Satsangi, G. S., Kumari, M., and Lakhani, A.: Study of size distribution of atmospheric aerosol at Agra, Atmos. Environ., 35, 693-702, 2001.

Pio, C. A. and Harrison, R. M.: Vapour pressure of ammonium chloride aerosol: effect of temperature and humidity, Atmos. Environ., 21, 2711-2715, 1987.

Pryor, S. C., Gallagher, M., Sievering, H., Larsen, S. E., Barthelmie, R. J., Birsan, F., Nemitz, E., Rinne, J., Kulmala, M., Grönholm, T., Taipale, R., and Vesala, T.: A review of measurement and modelling results of particle atmosphere-surface exchange, Tellus B, 60, 42-75, 2008.

Ruijgrok, W., Tieben, H., and Eisinga, P.: The dry deposition of particles to a forest canopy: A comparison of model and experimental results, Atmos. Environ., 31, 399-415, 1997.

Rupakheti, M., Leaitch, W. R., Lohmann, U., Hayden, K., Brickell, P., Lu, G., Li, S.-M., Toom-Sauntry, D., Bottenheim, J. W., Brook, J. R., Vet, R., Jayne, J. T., and Worsnop, R.: An intensive study of the size and composition of submicron atmospheric aerosols at a rural site in Ontario, Canada, Aerosol Sci. Technol., 39, 722-736, 2005.

Skeffington, R. A.: Quantifying uncertainty in critical loads: A literature review, Water Air Soil Pollut., 169, 3-24, 2006.

Trebs, I., Meixner, F. X., Slanina, J., Otjes, R., Jongejan, P., and Andreae, M. O.: Real-time measurements of ammonia, acidic trace gases and water-soluble inorganic aerosol species at a rural site in the Amazon Basin, Atmos. Chem. Phys., 4, 967-987, 2004, http://www.atmos-chem-phys.net/4/967/2004/.

Tsai, J.-H., Chang, K.-L., Lin, J.-J., Lin, Y.-H., and Chiang, H.L.: Mass-size distributions of particulate sulfate, nitrate, and ammonium in a particulate matter nonattainment region in southern Taiwan, J. Air Waste Manage. Assoc., 55, 502-509, 2005.
Tunved, P., Hansson, H.-C., Kulmala, M., Aalto, P., Viisanen, Y., Karlsson, H., Kristensson, A., Swietlicki, E., Dal Maso, M., Ström, J., and Komppula, M.: One year boundary layer aerosol size distribution data from five nordic background stations, Atmos. Chem. Phys., 3, 2183-2205, 2003, http://www.atmos-chem-phys.net/3/2183/2003/.

Van Dingenen, R., Putaud, J.-P., Martins-Dos Santos, S., and Raes, F.: Physical aerosol properties and their relation to air mass origin at Monte Cimone (Italy) during the first MINATROC campaign, Atmos. Chem. Phys., 5, 2203-2226, 2005, http://www.atmos-chem-phys.net/5/2203/2005/.

Vet, R., Brook, J. R., and Dann, T. F.: The nature of PM2.5 mass, composition, and precursors in Canada, in: Precursor Contributions to Ambient Fine Particulate Matter in Canada, chapter 4, a report by Environment Canada, available at: http://www. msc-smc.ec.gc.ca/saib/, 2001.

Wall, S. M., John, W., and Ondo, J. L.: Measurement of aerosol size distributions for nitrate and major ionic species, Atmos. Environ. 22, 1649-1656, 1988.

Watmough, S. A., Aherne, J., Alewell, C., Arp, P., Bailey, S., Clair, T., Dillon, P., Duchesne, L., Eimers, C., Fernandez, F., Foster, N., Larssen, T., Miller, E., Mitchell, M., and Page, S.: Sulphate, nitrogen and base cation budgets at 21 forested catchments in Canada, the United States and Europe, Environ. Monitor. Assess., 109, 1-36, 2005.

Whelpdale, D. M., Summers, P. W., and Sanhueza, E.: A global overview of atmospheric acid deposition fluxes, Environ. Monitor. Assess., 48, 217-247, 1997.

Winklmayr, W., Hwa-Chi, W., and John, W.: Adaptation of the Twomey algorithm to the inversion of cascade impactor data, Aerosol Sci. Technol., 13, 322-331, 1990.

Wolff, G. T.: On the nature of nitrate in coarse continental aerosols, Atmos. Environ., 18, 997-981, 1984.

Xiu, G., Zhang, D., Chen, J., Huang, Z., Chen, Z., Guo, H., and Pan, J.: Characterization of major water-soluble inorganic ions in size-fractionated particulate matters in Shanghai campus ambient air, Atmos. Environ., 38, 227-236, 2004.

Yao, X., Fang, M., and Chan, C. K.: The size dependence of chloride depletion in fine and coarse sea-salt particles, Atmos. Environ., 37, 743-751, 2003.

Yao, X., Ling, T. Y., Fang, M., and Chan, C. K.: Size dependence of in situ $\mathrm{pH}$ in submicron atmospheric particles in Hong Kong, Atmos. Environ., 41, 382-393, 2007.

Yoshizumi, K. and Hoshi, A.: Size distributions of ammonium nitrate and sodium nitrate in atmospheric aerosols, Environ. Sci. Technol., 19, 258-261, 1985.

Zhuang, H., Chan, C. K., Fang, M., and Wexler, A. S.: Size distribution of particulate sulfate, nitrate and ammonium at a coastal site in Hong Kong, Atmos. Environ., 33, 843-853, 1999. 